

Л. А. Саханенко, Асимптотика супремума взвешенных гауссовских полей с приложениями к ядерным оценкам плотностей, Теория вероятн. и ее примен., 2014, том 59, выпуск $3,499-541$

DOI: https://doi.org/10.4213/tvp4581

Использование Общероссийского математического портала Math-Net.Ru подразумевает, что вы прочитали и согласны с пользовательским соглашением http://www . mathnet.ru/rus/agreement

Параметры загрузки:

IP : 54.197 .217 .227

26 апреля 2023 г., $17: 11: 15$

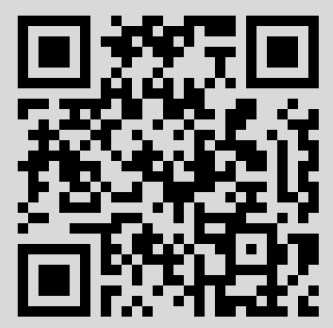




\section{АСИМПТОТИКА СУПРЕМУМА ВЗВЕШЕННЫХ ГАУССОВСКИХ ПОЛЕЙ С ПРИЛОЖЕНИЯМИ К ЯДЕРНЫМ ОЦЕНКАМ ПЛОТНОСТЕЙ ${ }^{1)}$}

Для центрированных стационарных гауссовских полей $X(s)$, $s \in \mathbf{R}^{d}, d>1$, мы показываем, что специальным образом центрированный и нормированный супремум $\sup _{s \in \mathbf{R}^{d}} w(s / T)|X(s)| \mathrm{c}$ неотрицательной весовой функцией $w$ при $T \rightarrow \infty$ сходится по распределению к двойному экспоненциальному закону, если $w$ и ковариационная функция процесса $X$ удовлетворяют определенным условиям гладкости и регулярности. Эта предельная теорема обобщает результаты для компактных множеств без весовой функции, полученные ранее в [11].

Затем этот новый результат для гауссовских полей применяется для нахождения необходимых и достаточных условий для сходимости по распределению к двойному экспоненциальному закону специальным образом центрированной и нормированной последовательности $\sup _{x \in \mathbf{R}^{d}}\left|\Psi(x)\left(\widehat{f}_{n}(x)-\mathbf{E} \widehat{f}_{n}(x)\right)\right|$ при естественных условиях гладкости (здесь $\widehat{f}_{n}$ обозначает ядерную оценку ограниченной непрерывной плотности $f$ на $\mathbf{R}^{d}$, построенную по выборке размера $n$, а $\Psi$ - положительная непрерывная функция такая, что $\sup _{x \in \mathbf{R}^{d}}\left|\Psi(x) f(x)^{\beta}\right|<\infty$ для некоторого $\left.\beta \in(0,1 / 2)\right)$.

Данная статья обобщает результаты недавней работы [4] на случай $d>1$. Она также обобщает результаты работы [12] для $\Psi(\cdot)=f^{-1 / 2}(\cdot) I_{S}(\cdot)$ с некоторым компактным множеством $S$ из $\mathbf{R}^{d}$ на общий класс функций $\Psi$. Пример практического применения завершает данную работу.

Ключевые слова и фразы: ядерные оценки плотностей, гауссовские процессы, взвешенная равномерная норма, локальный принцип инвариантности.

1. Введение. Пусть $X(s), s \in \mathbf{R}^{d}$, - центрированное стационарное гауссовское поле с ковариацией $\mathbf{E}\left[X\left(s_{1}\right) X\left(s_{2}\right)\right]=r\left(s_{1}-s_{2}\right)$, удовлетворяющей следующим условиям:

(R1) $r(s)=1-C_{r}\|s\|^{\alpha}+o\left(\|s\|^{\alpha}\right)$ при $\|s\| \rightarrow 0$ для некоторого $\alpha \in(0,2]$ и некоторого $C_{r}>0$;

(R2) $r(s)=0$ для всех $s$ таких, что $\|s\|>1$.

*Department of Statistics and Probability, Michigan State University, East Lansing, MI, USA; e-mail: luda@stt.msu.edu

1) Работа выполнена при поддержке NSF (грант DMS-0806176). 
Здесь и всюду ниже $\|x\|^{p}:=\sum_{i=1}^{d}\left|x_{i}\right|^{p}$ для $x=\left(x_{1}, \ldots, x_{d}\right) \in \mathbf{R}^{d}$ при некотором фиксированном действительном $p \geqslant 1$.

Заметим, что из однородности этого гауссовского поля и условия (R1) вытекает, что функция $r(s), s \in \mathbf{R}^{d}$, непрерывна всюду. Кроме того условия (R1) и (R2) влекут, что для всех $\varepsilon>0$ мы имеем $\sup _{\|s\|>\varepsilon}|r(s)|<1$. Заметим также, что по теореме 5 в [2, гл. IV.5] поле $X(s), s \in \mathbf{R}^{d}$, имеет сепарабельную версию с непрерывными траекториями, которую мы и будем использовать.

Для $d \geqslant 1$ и любого компактного множества $S$ из $\mathbf{R}^{d}$ В.И. Питербарг [11] получил точную асимптотику вероятностей $\mathbf{P}\left(\max _{s \in S} X(s)>\right.$ $u)$ и $\mathbf{P}\left(\max _{s \in S}|X(s)|>u\right)$ при $u \rightarrow \infty$. Результат для $d=1$ имеет длинную историю, начатую Дж. Пикандсом [10]. Все детали и полную библиографию см. в [11] и [9].

Нас интересует изучение супремума взвешенного гауссовского поля по всему пространству $\mathbf{R}^{d}$. На интуитивном уровне понятно, что по существу весовая функция контролирует поведение вне компактного множества. Обозначим весовую функцию через $w: \mathbf{R}^{d} \rightarrow \mathbf{R}_{+}$. Она удовлетворяет следующим трем условиям.

$($ w1) Носитель $W$ функции $w(x)$ состоит из конечного числа замкнутых прямоугольных параллелепипедов или квадрантов. Функция $w(x)$ непрерывна на $W$ и стремится к нулю при $\|x\| \rightarrow \infty$.

Для того чтобы сформулировать оставшиеся два условия, нам нужны дополнительные определения. Пусть

$$
\Phi_{d, \alpha}(u):=\frac{2}{\sqrt{2 \pi}} H_{\alpha} C_{r}^{1 / \alpha} u^{2 d / \alpha-1} \exp \left\{-\frac{u^{2}}{2}\right\}, \quad u>0, \quad \Phi_{d, \alpha}(\infty):=0,
$$

где $H_{\alpha}$ - константа Пикандса. Она может быть определена как предел

$$
H_{\alpha}=\lim _{M \rightarrow \infty} M^{-d} \mathbf{E} \exp \left\{\max _{x \in[0, M]^{d}} \chi(x)\right\}
$$

где $\chi(x), x \in \mathbf{R}^{d}$, - гауссовское поле с непрерывными траекториями, с функцией математического ожидания $\mathbf{E} \chi(x)=-\|x\|^{\alpha}$ и ковариационной функцией $r_{\chi}(x, y)=\|x\|^{\alpha}+\|y\|^{\alpha}-\|x-y\|^{\alpha}$. Также определим

$$
\Lambda_{d, \alpha}(u):=\int_{W} \Phi_{d, \alpha}\left(\frac{u}{w(y)}\right) d y, \quad u>0 .
$$

Тогда весовая функция $w$ удовлетворяет также следующим условиям:

(w2) существует $u_{0} \in(0, \infty)$ такое, что $\Lambda_{d, \alpha}\left(u_{0}\right)<\infty$;

(w3) при $T \rightarrow \infty$

$$
\sup _{s, s+\tau \in W,\|\tau\| \leqslant 1 / T}\left|\frac{w(s+\tau)}{w(s)}-1\right|=o\left(\frac{1}{\ln T}\right) .
$$


Заметим, что из условий $(\mathrm{w} 1)$ и $(\mathrm{w} 2)$ следует, что $\Lambda_{d, \alpha}(u)-$ монотонно убывающая к 0 функция при $u \rightarrow \infty$, начиная с достаточно большого $u>0$.

Пусть $A_{T}$ - решение уравнения

$$
T^{d} \Lambda_{d, \alpha}\left(A_{T}\right)=1
$$

Из условий (w1) и (w2) следует, что последовательность $A_{T}$ существует и единственна для всех достаточно больших $T$. Отметим также, что $A_{T} \rightarrow \infty$ при $T \rightarrow \infty$. Более того, по лемме 5 в [4] мы имеем

$$
0<\liminf _{T \rightarrow \infty} \frac{A_{T}}{\sqrt{\ln T}} \leqslant \limsup _{T \rightarrow \infty} \frac{A_{T}}{\sqrt{\ln T}}<\infty .
$$

На самом деле верно более сильное утверждение.

Предложение 1. Пусть выполненьл условия (w1)-(w3). Тогда при $T \rightarrow \infty$

$$
A_{T}=\sup _{x \in \mathbf{R}^{d}} w(x) \sqrt{2 d \ln T}+\left(\frac{d}{\alpha}-\frac{1}{2}\right) \sup _{x \in \mathbf{R}^{d}} w(x) \frac{\ln \ln T}{\sqrt{2 d \ln T}}+o\left(\frac{1}{\sqrt{\ln T}}\right) .
$$

Теперь мы можем сформулировать главный результат для супремума взвешенного стационарного гауссовского поля.

Теорема 1. Пусть выпполнень условия (R1), (R2) и (w1)-(w3). Для всех $t \in \mathbf{R}$ мьи имеем при $T \rightarrow \infty$

$$
\mathbf{P}\left(A_{T}\left(\sup _{s \in \mathbf{R}^{d}} w\left(\frac{s}{T}\right)|X(s)|-A_{T}\right) \leqslant t\right) \rightarrow \exp \left\{-e^{-t}\right\} .
$$

Данная работа о гауссовских полях мотивирована следующей задачей оценивания плотности. Пусть $X_{1}, \ldots, X_{n}$ - независимые одинаково распределенные наблюдения с неизвестной плотностью $f: \mathbf{R}^{d} \rightarrow \mathbf{R}$, где $d>1$. Определим ядерную оценку для плотности $f$ на основе данных $\left(X_{1}, \ldots, X_{n}\right)$ с ядром $K$ формулой

$$
\widehat{f}_{n}(x)=\frac{1}{n h_{n}^{d}} \sum_{i=1}^{n} K\left(\frac{x-X_{i}}{h_{n}}\right), \quad x \in \mathbf{R}^{d},
$$

где ширина окна $h_{n}$ удовлетворяет условиям $h_{n} \rightarrow 0$ и $n h_{n}^{d} \rightarrow \infty$ при $n \rightarrow \infty$, которые влекут за собой состоятельность оценки $\widehat{f}_{n}$.

Пусть $\Psi: \mathbf{R}^{d} \rightarrow \mathbf{R}$ - положительная непрерывная функция. Э. Жине, В.Колчинский и Дж. Зинн в [5] показали, что при $n \rightarrow \infty$ последовательность

$$
\sqrt{\frac{n h_{n}^{d}}{2\left|\ln h_{n}^{d}\right|}}\left\|\Psi(\cdot)\left(\widehat{f}_{n}(\cdot)-\mathbf{E} \widehat{f}_{n}(\cdot)\right)\right\|_{\infty}
$$


сходится почти наверное к константе, если выполнены определенные условия гладкости и регулярности на $f, \Psi, K$ и функцию ширины окна $h_{t}, t \geqslant 1$, включающие в себя, в частности, предположения $\sup _{x \in \mathbf{R}^{d}}\left|\Psi(x) f^{\beta}(x)\right|=\left\|\Psi f^{\beta}\right\|_{\infty}<\infty$ для некоторого $\beta \in(0,1 / 2)$ и $\int_{1}^{\infty} \mathbf{P}\left(\Psi\left(X_{1}\right)>\left(t h_{t}^{d}\left|\ln h_{t}\right|\right)^{1 / 2}\right) d t<\infty$.

Одна из целей данной работы - изучить сходимость по распределению последовательности (2) при надлежащих центрировании и нормировке. Эта статья продолжает линию исследований, начатую В. Д. Бикелем и М. Розенблаттом [1], которые рассмотрели задачи подобного характера с $\Psi=f^{-1 / 2}$ на ограниченном интервале действительной прямой. Позже В. Д. Конаков и В. И. Питербарг [7] изучали сходимость по распределению взвешенной равномерной нормы разности между ядерной оценкой регрессионной функции и ее математическим ожиданием на компактном множестве. Используя некоторые их результаты для гауссовских полей и новый локальный принцип инвариантности, Э. Рио [12] доказал сходимость по распределению взвешенной равномерной нормы разности между ядерной оценкой плотности и ее математическим ожиданием для случая $\Psi=f^{-1 / 2}$ на компактном множестве в многомерной ситуации $d>1$. Недавно Э. Жине, В. Колчинский и Л. Саханенко [4] доказали сходимость по распределению последовательности (2) к повторному экспоненциальному закону в одномерной ситуации $d=1$.

Но их доказательство специфично для одномерного случая. Для многомерного случая нужен иной подход. В настоящей статье мы отвечаем на эту потребность. Мы также раздвигаем рамки исследований скоростей сходимости взвешенных равномерных норм разности между ядерной оценкой плотности и ее математическим ожиданием. Мы получаем скорости сходимости по распределению, тогда как в [5] получены скорости сходимости по вероятности и почти наверное. При определенных условиях гладкости и регулярности на $f, \Psi, K$ и $h_{n}$ мы показываем, что, в зависимости от выбранных ширины окна $h_{n}$, центрирующей и нормирующей последовательностей, возможен один из следующих трех случаев:

(I) последовательность

$$
\sqrt{\frac{0.5 n h_{n}^{d}}{\left|\ln h_{n}\right|}}\left\|\Psi(\cdot)\left(\widehat{f}_{n}(\cdot)-\mathbf{E} \widehat{f}_{n}(\cdot)\right)\right\|_{\infty}
$$

сходится по распределению к обрезанной неотрицательной случайной величине с кумулятивной функцией распределения типа $\exp \left\{-t^{\delta}\right\}$ для конкретного $\delta>0$;

(II) для конкретной регулярно меняющейся функции $d_{t}$ последовательность

$$
\frac{n h_{n}^{d}}{d_{n}}\left\|\Psi(\cdot)\left(\widehat{f}_{n}(\cdot)-\mathbf{E} \widehat{f}_{n}(\cdot)\right)\right\|_{\infty}
$$


сходится по распределению к неотрицательной случайной величине с кумулятивной функцией распределения типа $\exp \left\{-t^{\delta}\right\}$ для конкретного $\delta>0$

(III) для последовательности $A_{1 / h_{n}}$, являющейся $A_{T}$ с $T=1 / h_{n}$, и $w=\Psi f^{1 / 2}$ последовательность

$$
A_{1 / h_{n}}\left(\frac{\sqrt{n h_{n}^{d}}}{\|K\|_{2}}\left\|\Psi(\cdot)\left(\widehat{f}_{n}(\cdot)-\mathbf{E} \widehat{f}_{n}(\cdot)\right)\right\|_{\infty}-A_{1 / h_{n}}\right),
$$

где $\|K\|_{2}^{2}=\int_{\mathbf{R}^{d}} K^{2}(x) d x$, сходится по распределению к двойной экспоненциальной случайной величине.

Сходимость (III) - изящная, она связана с теоремой 1 через локальное сильное приближение в смысле [12]. Точнее, для центрированного стационарного гауссовского поля $X(y), y \in \mathbf{R}^{d}$, с ковариационной функцией $r(y)=\int_{\mathbf{R}^{d}} K(y+s) K(s) d s /\|K\|_{2}$ мы покажем (см. теорему 4), что последовательности

$$
\left\{A_{1 / h_{n}}\left(\frac{\sqrt{n h_{n}^{d}}}{\|K\|_{2}} \sup _{t \in D_{a}}\left|\Psi(t)\left(\widehat{f}_{n}(t)-\mathbf{E} \widehat{f}_{n}(t)\right)\right|-A_{1 / h_{n}}\right)\right\}
$$

и

$$
\left\{A_{1 / h_{n}}\left(\sup _{t \in D_{a}}\left|\Psi(t) \sqrt{f(t)} X\left(\frac{t}{h_{n}}\right)\right|-A_{1 / h_{n}}\right)\right\}
$$

сходятся или расходятся по распределению вместе при $n \rightarrow \infty$ для любых достаточно больших $a>0$, где $D_{a}=\left\{x \in \mathbf{R}^{d}:\|x\| \leqslant a, f(x) \geqslant 1 / a\right\}$. Теорема 1 применяется к процессу $X(y), y \in \mathbf{R}^{d}$, из чего вытекает сходимость специальным образом центрированной и нормированной последовательности (2) к двойному экспоненциальному распределению.

Оставшаяся часть статьи организована следующем образом. Раздел 2 посвящен обозначениям, условиям и основным результатам для оценок плотностей. Раздел 3 связывает наш асимптотический результат для супремума взвешенных стационарных гауссовских полей с нашей задачей оценивания плотности через локальное сильное приближение в смысле Я. Комлоша, П. Майора и Г. Тушнади [6] (KMT), используя подход [12].

Доказательство теоремы 1 приведено в разделе 4. В разделе 5 доказываются теоремы 2 и 3 о скорости сходимости по распределению для специальным образом нормированной и центрированной последовательности (2). Пример практического применения в разделе 6 завершает эту работу.

\section{2. Условия и основные результаты для оценок плотностей.} Мы используем условия, требующиеся в [5]. По-видимому, они являются необходимыми, когда мы имеем дело с взвешенными равномерными нормами по всему пространству $\mathbf{R}^{d}$. См. упомянутую выше статью, где 
обсуждаются эти условия и приведен ряд примеров, иллюстрирующих их необходимость. Мы формулируем эти условия ниже для полноты.

(K1) $K$ - ограниченная измеримая функция плотности вероятностей с носителем, лежащим в $[-1 / 2,1 / 2]^{d}$, которая принадлежит линейной оболочке, натянутой на функции $\kappa \geqslant 0$, обладающие следующим свойством: подграф $\kappa$ (т.е. множество вида $\{(s, u): \kappa(s) \geqslant u\}$ ) может быть представлен как результат применения конечного числа булевых операций к множествам вида $\{(s, u): p(s, u) \geqslant q(u)\}$, где $p$ - полином на $\mathbf{R}^{d} \times \mathbf{R}$, а $q$ - произвольная действительная функция. В частности, отсюда следует, что у $K$ ограниченная вариация.

(К2) Функция

$$
r(x)=\frac{1}{\|K\|_{2}} \int_{\mathbf{R}^{d}} K(x+s) K(s) d s, \quad x \in \mathbf{R}^{d},
$$

удовлетворяет условиям (R1), (R2) из введения.

(K3) $K$ - дифференцируемая функция такая, что

$$
\int\left|\sum_{i=1}^{d} \frac{\partial K}{\partial z_{i}}(z)\right| d z=C_{K}<\infty .
$$

Условие (K2) на $K$ не требовалось в [5], но требуется в [4]. Условие (К3) ново и специфично для нашего подхода.

Плотность $f$ и весовая функция $\Psi$ удовлетворяют приведенным в $[5]$ условиям (D.a)-(D.c), (W.a)-(W.c), (WD.a) $\beta$ для некоторого $0<\beta<2$ и условиям $\left(\mathrm{H}_{1}\right),\left(\mathrm{H}_{2}\right)$. Мы формулируем их ниже для полноты.

(D.a) $f$ - ограниченная плотность на $\mathbf{R}^{d}$, непрерывная на множестве $B_{f}:=\left\{x \in \mathbf{R}^{d}: f(x)>0\right\}$, где она положительна; предполагается также, что $B_{f}$ есть замыкание открытого множества и $\lim _{a \rightarrow \infty} \sup _{\|x\|>a} f(x)=0$.

(D.b) Для любого $\delta>0$ существуют $c>0$ и $h_{0}>0$ такие, что при всех $\|y\| \leqslant h_{0}$ и $x \in B_{f}$, для которых $x+y \in B_{f}$, выполнены неравенства

$$
\frac{1}{c} f^{\delta}(x) \leqslant \frac{f(x+y)}{f(x)} \leqslant c f^{-\delta}(x) .
$$

(D.c) Для всех $\rho>0$

$$
\lim _{h \rightarrow 0} \sup _{x, y: f(x) \geqslant h^{\rho}, x+y \in B_{f},\|y\| \leqslant h}\left|\frac{f(x+y)}{f(x)}-1\right|=0 .
$$

(W.a) $\Psi: B_{f} \rightarrow \mathbf{R}_{+}$- положительная непрерывная функция на $B_{f}$.

(W.b) Для любого $\delta>0$ существуют $c>0$ и $h_{0}>0$ такие, что при всех $\|y\| \leqslant h_{0}$ и $x \in B_{f}$, для которых $x+y \in B_{f}$, выполнены неравенства

$$
\frac{1}{c} \Psi^{-\delta}(x) \leqslant \frac{\Psi(x+y)}{\Psi(x)} \leqslant c \Psi^{\delta}(x) .
$$


(W.c) Для всех $\rho>0$

$$
\lim _{h \rightarrow 0} \sup _{x, y: \Psi(x) \leqslant h^{-\rho}, x+y \in B_{f},\|y\| \leqslant h}\left|\frac{\Psi(x+y)}{\Psi(x)}-1\right|=0 .
$$

(WD.a) $\sup _{x \in B_{f}}\left|\Psi(x) f^{\beta}(x)\right|<\infty$, где $\beta \in(0,2)$.

(H1) Функция ширины окна $h_{t}=h(t), t \geqslant 1$, монотонно убывает к 0 , а функция $t h_{t}^{d}$ - строго возрастает к бесконечности при $t \rightarrow \infty$.

$(\mathrm{H} 2) h_{t}^{d}$ регулярно меняется на бесконечности с показателем $-\eta$ для некоторого $\eta \in(0,1)$.

Нам также требуются следующие условия из [4].

(F1) Множество $B_{f}$ является конечным объединением замкнутых прямоугольных параллелепипедов и квадрантов. Более того, $f$ удовлетворяет на $B_{f}$ условию Гёльдера с некоторым показателем $\gamma>0$.

(F2) Функция $w=\Psi f^{1 / 2}$ удовлетворяет приведенным во введении условиям (w1)-(w3).

Теперь мы готовы сформулировать главный результат этого раздела.

Теорема 2. Пусть выполнень условия (K1)-(K3), (D.a)-(D.c), (W.a)-(W.c), (H1)-(H2), (WD.a) $)_{\beta}$, (F1)-(F2). Предположим, что либо $B_{f}=\mathbf{R}^{d}$, либо $K(0)=\|K\|_{\infty} u \beta \in(0,1 / 2)$. Тогда для всех $t \in \mathbf{R}$ мь имеем

$$
\mathbf{P}\left(A_{1 / h_{n}}\left(\frac{\sqrt{n h_{n}^{d}}}{\|K\|_{2}}\left\|\Psi(\cdot)\left(\widehat{f}_{n}(\cdot)-\mathbf{E} \widehat{f}_{n}(\cdot)\right)\right\|_{\infty}-A_{1 / h_{n}}\right) \leqslant t\right) \rightarrow \exp \left\{-e^{-t}\right\}
$$

при $n \rightarrow \infty$ в том и только том случае, когда

$$
\lim _{t \rightarrow \infty} t \mathbf{P}\left(\Psi\left(X_{1}\right)>\left(t h_{t}^{d}\left|\ln h_{t}\right|\right)^{1 / 2}\right)=0 .
$$

Для случая $\Psi(\cdot)=\|f\|_{\infty}^{-1 / 2} I\left(\cdot \in B_{f}\right)$ мы имеем следующим результат.

Следствие 1. Пусть выполнень условия (K1)-(K3), (F1). Предположим, что $K(0)=\|K\|_{\infty}$, если $B_{f} \neq \mathbf{R}^{d}$. Пусть $h_{n} \searrow 0, n h_{n}^{d} \nearrow \infty$, $n h_{h}^{d} /\left(\left|\ln h_{n}\right|(\ln n)^{2}\right) \rightarrow \infty u\left|\ln h_{n}\right| / \ln \ln n \rightarrow \infty$ при $n \rightarrow \infty$. Также предположим, ито для каждого $а>0$ и всех достаточно больших $T>0$

$$
\sup _{x, y \in D_{a},\|x-y\|<1 / T}\left|1-\sqrt{\frac{f(x)}{f(y)}}\right|=o\left(\frac{1}{\ln T}\right) .
$$

Тогда для всех $t \in \mathbf{R}$ мьи имеем

$$
\mathbf{P}\left(A_{1 / h_{n}}\left(\frac{\sqrt{n h_{n}^{d}}}{\|K\|_{2}\|f\|_{\infty}^{1 / 2}}\left\|\widehat{f}_{n}(\cdot)-\mathbf{E} \widehat{f}_{n}(\cdot)\right\|_{\infty}-A_{1 / h_{n}}\right) \leqslant t\right) \rightarrow \exp \left\{-e^{-t}\right\},
$$

где $h_{n}^{-d} \Lambda_{d, \alpha}\left(A_{1 / h_{n}}\right)=1$ c w $(\cdot)=f^{1 / 2}(\cdot)\|f\|_{\infty}^{-1 / 2} I\left(\cdot \in B_{f}\right)$. 
Это многомерная версия теоремы 7 в [4]. Следующая теорема - это многомерная версия теорем 8 и 9 в [4]. Напомним, что $\eta$ - показатель регулярно меняющейся функции $h_{t}$.

Теорема 3. Предположим, что выполнень условия теоремь 1. Если $\beta \in(0,1 / 2)$, то существование предела

$$
\lim _{t \rightarrow \infty} t \mathbf{P}\left(\Psi\left(X_{1}\right)>\left(t h_{t}^{d}\left|\ln h_{t}\right|\right)^{1 / 2}\right)=L \in[0, \infty)
$$

является необходимьм и достаточным условием для сходимости

$\sqrt{\frac{0.5 n h_{n}^{d}}{\left|\ln h_{n}\right|}}\left\|\Psi(\cdot)\left(\widehat{f}_{n}(\cdot)-\mathbf{E} \widehat{f}_{n}(\cdot)\right)\right\|_{\infty} \stackrel{d}{\longrightarrow}\left(2^{-1 / 2}\|K\|_{\infty} Z_{\eta, L}\right) \vee\left(\|K\|_{2}\left\|\Psi f^{1 / 2}\right\|_{\infty}\right) ;$ если же $\beta \in(0,1]$, то существование предела

$$
\lim _{t \rightarrow \infty} t \mathbf{P}\left(\Psi\left(X_{1}\right)>d_{t}\right)=L \in[0, \infty)
$$

для некоторой строго возрастающей регулярно меняющейся функции $d_{t}$ с показателем $\eta_{1}$ таким, ито $d_{t} /\left(t h_{t}^{d}\left|\ln h_{t}\right|\right)^{1 / 2} \rightarrow \infty u d_{t} \geqslant c t^{\beta} \partial \Omega s$ некоторого с > 0, является необходимим и достаточным условием для сходимости:

$$
\frac{n h_{n}^{d}}{d_{n}}\left\|\Psi(\cdot)\left(\widehat{f}_{n}(\cdot)-\mathbf{E} \widehat{f}_{n}(\cdot)\right)\right\|_{\infty} \stackrel{d}{\longrightarrow}\|K\|_{\infty} Z_{\eta_{1}, L}
$$

где кумулятивная функиия распределения неотрииательной случайной величинь $Z_{\eta, L}$ равна $\exp \left\{-L t^{-2 /(1-\eta)}\right\}$ при $t>0$ и $Z_{\eta, 0}=0$.

Теоремы 2 и 3 описывают единственные случаи, когда специальным образом центрированная и нормированная норма $\left\|\Psi(\cdot)\left(\widehat{f}_{n}(\cdot)-\mathbf{E} \widehat{f}_{n}(\cdot)\right)\right\|_{\infty}$ сходится к невырожденному распределению.

3. Гауссовское приближение. В этом разделе $X(y), y \in \mathbf{R}^{d}$, будет стационарным центрированным гауссовским полем с ковариационной функцией

$$
r(y)=\frac{1}{\|K\|_{2}} \int_{\mathbf{R}^{d}} K(y+s) K(s) d s, \quad y \in \mathbf{R}^{d} .
$$

Напомним, что $D_{a}=\left\{x \in \mathbf{R}^{d}:\|x\| \leqslant a, f(x) \geqslant 1 / a\right\}$ для $a>0$. В этом случае мы имеем следующий результат о сильном приближении.

Теорема 4. Предположим, что вьлолнень условия (K1)-(K3), (H1)-(H2), (D.a), (D.c), (F1) и (W.a). Также будем считать, что $\Psi$ ограничена на $B_{f}$. Предположим, что $\alpha_{n} \nearrow \infty, n h_{n}^{d+1} \nearrow C>0$ или $n h_{n}^{d+1} \nearrow \infty u$

$$
\begin{gathered}
\alpha_{n}=o\left(h_{n}^{-\gamma / 2}\left|\ln h_{n}\right|^{-1 / 2} \wedge\left(n h_{n}^{d+1}\right)^{1 / 2} \ln n \wedge\left(n^{1 / d} h_{n} \ln n\right)^{1 / 2} \wedge h_{n}^{-d / 2}\right. \\
\left.\wedge \frac{n^{1 / 2}}{\ln n} \wedge \frac{\left(n h_{n}^{d}\right)^{1 / 2}}{\ln n} \wedge \sqrt{\frac{n h_{n}^{-d / 2}}{\ln n}}\right) .
\end{gathered}
$$


при $n \rightarrow \infty$. Тогда для любого достаточно большого а $>0$ две последовательности

$$
\left\{\alpha_{n}\left(\frac{\sqrt{n h_{n}^{d}}}{\|K\|_{2}} \sup _{x \in D_{a}}\left|\Psi(x)\left(\widehat{f}_{n}(x)-\mathbf{E} \widehat{f}_{n}(x)\right)\right|-\alpha_{n}\right)\right\}
$$

$u$

$$
\left\{\alpha_{n}\left(\sup _{y \in D_{a}}\left|\Psi(y) \sqrt{f(y)} X\left(\frac{y}{h_{n}}\right)\right|-\alpha_{n}\right)\right\}
$$

одновременно сходятся или расходятся по распределению при $n \rightarrow \infty$.

3 а м е ч а н и е. Из условий (H1)-(H2) и (1) очевидно, что теорема 4 работает с $\alpha_{n}=A_{1 / h_{n}}$.

Д о к а з а т е л ь с т в о. Мы комбинируем результаты работы [12] о локальном принципе инвариантности с подходом, похожим на доказательство в [4, п. 2].

Мы начнем с формулировки леммы, которая слегка модифицирует теорему 1.1 в [12]. Пусть $Y_{1}, \ldots, Y_{n}$ независимые одинаково распределенные случайные величины, имеющие вероятностное распределение $Q_{0}$ с плотностью, непрерывной и строго положительной на замкнутом прямоугольном параллелепипеде $\mathscr{R}$ из $\mathbf{R}^{d}$. Обозначим

$$
\nu_{n}=n^{-1 / 2} \sum_{i=1}^{n}\left(\delta_{Y_{i}}-Q_{0}\right)
$$

Пусть $\mathscr{S}$ - это класс функций, отображающих $\mathscr{R}$ в $[-1,1]$. Для вероятностного закона $Q$ на $\mathscr{R}$ и $\varepsilon \in(0,1)$ обозначим наибольшую мощность $\varepsilon$-разделенной сети для $\mathscr{S}$ в $L_{1}(Q)$ через $N(\varepsilon, \mathscr{S}, Q)$. Теперь обозначим $N(\varepsilon, \mathscr{S})=\sup _{Q} N(\varepsilon, \mathscr{S}, Q)$, где супремум берется по всем вероятностным законам $Q$ на $\mathscr{R}$ с конечным носителем. Если

$$
N(\varepsilon, \mathscr{S}) \leqslant C(\mathscr{S}) \varepsilon^{-d(\mathscr{S})} \quad \forall \varepsilon \in(0,1)
$$

с некоторыми положительными константами $C(\mathscr{S})$ и $d(\mathscr{S})$, то $\mathscr{S}$ называется классом функций Вапника-Червоненкиса. Подробности об энтропийных числах, классах Вапника-Червоненкиса и их свойствах см. [13].

Для функции $g \in \mathscr{S}$ обозначим

$$
K(g, \mathscr{R})=\sup _{v} \frac{1}{\|v\|_{\infty}}\left|\int_{\mathbf{R}^{d}} g(x) \sum_{i=1}^{d} \frac{\partial v}{\partial x_{i}} d x\right|,
$$

где супремум берется по всем бесконечно дифференцируемым функциям $v: \mathscr{R} \rightarrow \mathbf{R}^{d}$ с компактным носителем в $\mathscr{R}$ и $\|v\|_{\infty}=\sup _{x \in \mathbf{R}^{d}}\|v(x)\|_{2}$. Класс $\mathscr{S}$ удовлетворяет следующим условиям:

$$
(\mathrm{UBV}) K(\mathscr{S}):=\sup _{g \in \mathscr{S}} K(g, \mathscr{R})<\infty
$$


(LUBV) для любого $a \in(0,1)$, любого прямоугольного параллелепипеда $\mathscr{C} \subset \mathscr{R}$, а раз пропорционального $\mathscr{R}$ с ребрами, параллельными ребрам $\mathscr{R}$, и любой $g \in \mathscr{S}$ выполнено неравенство $K(g, \mathscr{C}) \leqslant K_{\text {lос }}(\mathscr{S}) a^{d-1}$ для некоторой константы $K_{\text {lос }}(\mathscr{S}) \geqslant 1$, зависящей только от $\mathscr{S}$;

(M) существуют пространство Суслина $V$ и отображение $\mathscr{T}$ из $V$ на $\mathscr{S}$ такие, что $(x, y) \mapsto \mathscr{T}(y) x$ измеримо на $\mathbf{R}^{d} \times V$.

Говорят, что у $\mathscr{S}$ равномерно ограниченная вариация, если выполнено (UBV). Исходя из таких же соображений, говорят, что у $\mathscr{S}$ локально равномерно ограниченная вариация, если выполнено (LUBV).

Всюду далее $B_{n, Q_{0}}$ обозначает броуновский мост, индексированный функциями из $\mathscr{S}$, с почти наверное непрерывными траекториями на $(\mathscr{S}, P)$, т.е. центрированное гауссовское поле с ковариационной функцией

$$
\left(g_{1}, g_{2}\right) \rightarrow \mathbf{E}_{Q_{0}}\left(g_{1}\left(Y_{1}\right) g_{2}\left(Y_{1}\right)\right)-\mathbf{E}_{Q_{0}}\left(g_{1}\left(Y_{1}\right)\right) \mathbf{E}_{Q_{0}}\left(g_{2}\left(Y_{1}\right)\right) .
$$

Предполагается, что вероятностное пространство достаточно богато.

Лемма 1. Пусть $d \geqslant 2$ и $Q_{0}$ - сосредоточенное на замкнутом прямоугольном параллелепипеде $\mathscr{R}$ вероятностное распределение с непрерьвной строго положительной плотностью. Пусть $\mathscr{S}$ будет классом функиий Вапника-Червоненкиса из $\mathscr{R}$ в $[-1,1]$, удовлетворяющим условию измеримости (M), условию (UBV) с некоторой константой $K(\mathscr{S})$ и энтропийному условию (3) с некоторыли положительными константами $C(\mathscr{S})$ и d(S). Тогда для каждого $n \geqslant 1$ существует броуновский мост $B_{n, Q_{0}}$, индексированньй функииями из $\mathscr{S}$, с почти наверное непрерьвныли траекториями на $(\mathscr{S}, P)$ такой, что для любыл $t \geqslant C \ln n$

$$
\mathbf{P}\left(\sqrt{n} \sup _{g \in \mathscr{S}}\left|\nu_{n}(g)-B_{n, Q_{0}}(g)\right| \geqslant C \sqrt{n^{(d-1) / d} K(\mathscr{S}) t}+C c_{n} t\right) \leqslant e^{-t},
$$

где $c_{n}=\sqrt{\ln n}$ в общем случае, $c_{n}=\sqrt{K_{\text {lос }}(\mathscr{S})}$ при условии (LUBV), $а$ $C$ - положительная константа, зависящая от $d, d(\mathscr{S})$ и $C(\mathscr{S})$.

Д о к а з а т е л ь с т в о. Заметим, что любое аффинное преобразование $\mathscr{T}: \mathbf{R}^{d} \rightarrow \mathbf{R}^{d}$, переводящее $\mathscr{R}$ в $[0,1]^{d}$, является диффеоморфизмом. Следовательно, $\mathscr{T}\left(Y_{1}\right), \ldots, \mathscr{T}\left(Y_{n}\right)$ - независимые одинаково распределенные случайные величины, имеющие распределение с непрерывной строго положительной на $[0,1]^{d}$ плотностью. Применение теоремы 1.1 из [12] дает требуемое утверждение.

3 а м е ч а н и е. Лемма 1 остается верной, если вместо замкнутого прямоугольного параллелепипеда $\mathscr{R}$ использовать замкнутое множество в $\mathbf{R}^{d}$, диффеоморфное кубу $[0,1]^{d}$.

Приведенная лемма лежит в основе доказательства теоремы 4. Однако по условию (F1) множество $B_{f}$, где плотность $f$ положительна, 
является объединением замкнутых прямоугольных параллелепипедов и квадрантов, что не позволяет нам применить лемму напрямую. Естественно, мы обрезаем $B_{f}$ на бесконечностях и разбиваем то, что осталось, на прямоугольные параллелепипеды, где лемма 1 применима. Мы сталкиваемся с дополнительными трудностями, которые преодолеваем с помощью классического результата Я. Комлоша, П. Майора и Г. Тушнади и стандартных неравенств на хвосты эмпирических и гауссовских процессов. Далее следуют технические детали. Для простоты изложения они поделены на несколько шагов.

1. Для фиксированного $a>0$ определим классы функций

$$
\begin{aligned}
\widetilde{\mathscr{F}}_{b_{1}, b_{2}} & =\left\{\Psi(x) K\left(\frac{x-\cdot}{h}\right): x \in D_{a}, b_{1} \leqslant h \leqslant b_{2}\right\}, \quad b_{2} \geqslant b_{1}>0, \\
\widetilde{\mathscr{F}}_{n} & :=\widetilde{\mathscr{F}}_{h_{n} / 2,2 h_{n}}=\left\{\Psi(x) K\left(\frac{x-\cdot}{h}\right): x \in D_{a}, \frac{h_{n}}{2} \leqslant h \leqslant 2 h_{n}\right\} .
\end{aligned}
$$

Пусть $x \in D_{a}$, тогда функция $K((x-t) / h), t \in \mathbf{R}^{d}$, может быть отлична от нуля только для таких $t$, что $\left|x_{i}-t_{i}\right| \leqslant 1 / 2, i=1, \ldots, d$. Таким образом, носитель любой функции $g \in \widetilde{\mathscr{F}}_{n}$ вкладывается в $\{t \in$ $\left.\mathbf{R}^{d}:\|t\|^{2} \leqslant 2 a^{2}+2 d h_{n}^{2}\right\}$. В силу условия (D.b) при данном $\delta=1$ существуют $c>0$ и $h_{0}>0$ такие, что $f(t) / f(x) \geqslant f(x) / c$, если $\sqrt{d} h_{n} \leqslant h_{0}$, и $f(t) \geqslant 1 /\left(c a^{2}\right)$. Таким образом, если $c^{2} a^{4} \geqslant 2 a^{2}+2 h_{0}$ и $n$ достаточно большое, то носитель любой функции $g \in \widetilde{\mathscr{F}}_{n}$ вкладывается в множество $D_{c a^{2}}$.

Заметим, что из-за условия (F1) множество $D_{c a^{2}}$ можно покрыть объединением конечного числа непересекающихся прямоугольных параллелепипедов, лежащих в $B_{f}$, которые мы обозначим через $B_{m}, m=$ $1, \ldots, M$. Пусть $B_{0}=D_{c a^{2}}^{c}$.

2. Для $m=0, \ldots, M$ определим $n_{m}$ как число попавших в $B_{m}$ случайных величин $X_{1}, \ldots, X_{n}$, которые перенумерованы и переобозначены как $Y_{i}^{m} \in B_{m}, i=1, \ldots, n_{m}, m=0, \ldots, M$. Пусть $p_{m}=P\left(B_{m}\right)$, $m=0, \ldots, M$. Введем случайную величину $Z:=\left(n_{0}, \ldots, n_{M}\right)$. Заметим, что она имеет полиномиальное распределение с параметрами $\left(n, p_{0}, \ldots, p_{M}\right)$. Более того, отметим, что для каждого $m=0, \ldots, M$ случайные величины $Y_{i}^{m}, i=1, \ldots, n_{m}$, - независимы и одинаково распределены на $B_{m}$ с распределением $P_{m}$. Кроме того векторы $\left(Y_{1}^{m}, \ldots, Y_{n_{m}}^{m}\right)$, $m=0, \ldots, M$, независимы. По построению для любой функции $g \in \widetilde{\mathscr{F}}_{n}$ мы имеем

$$
P_{m} g=\int g(y) d P_{m}(y)=\frac{1}{p_{m}} \int_{B_{m}} g(y) d P(y), \quad m=0, \ldots, M .
$$

Определим

$$
\nu_{n, P}=n^{-1 / 2} \sum_{i=1}^{n}\left(\delta_{X_{i}}-P\right)
$$




$$
\widetilde{\nu}_{n_{m}, P_{m}}=n_{m}^{-1 / 2} \sum_{i=1}^{n_{m}}\left(\delta_{Y_{i}^{m}}-P_{m}\right), \quad m=0, \ldots, M .
$$

Тогда условно на $Z=\left(n_{0}, \ldots, n_{M}\right)$ для любой функции $g \in \widetilde{\mathscr{F}}_{n}$ мы имеем следующее представление:

$$
\begin{aligned}
\sqrt{n} \nu_{n, P}(g) & =\sum_{i=1}^{n} g\left(X_{i}\right)-n P g=\sum_{i=1}^{n} \sum_{m=0}^{M} g\left(X_{i}\right) I_{B_{m}}\left(X_{i}\right)-\sum_{m=0}^{M} n P\left(g I_{B_{m}}\right) \\
& =\sum_{m=0}^{M}\left(\sum_{i=1}^{n} g\left(X_{i}\right) I_{B_{m}}\left(X_{i}\right)-n p_{m} P_{m} g\right)+\sum_{m=0}^{M}\left(n_{m}-n p_{m}\right) P_{m} g \\
& =\sum_{m=0}^{M}\left[\sqrt{n_{m}} \nu_{n_{m}, P_{m}}(g)+\left(n_{m}-n p_{m}\right) P_{m} g\right] .
\end{aligned}
$$

3. Для $m=1, \ldots, M$ мы можем применить лемму 1 , поскольку плотность $f$ строго положительна на замыкании $B_{m}$ и класс $\widetilde{\mathscr{F}}_{n}$ удовлетворяет условиям (M), (UBV) и $(\mathrm{LUBV})$. То, что $\widetilde{\mathscr{F}}_{n}$ удовлетворяет условию измеримости (M), очевидно. Более того, он является классом Вапника-Червоненкиса в силу условия $(\mathrm{K})$ и равномерной ограниченности функции $\Psi$ на $D_{a}$. Тогда для всех вероятностных мер $Q$ (см. в $[5$, c. 2573])

$$
N\left(\varepsilon, \widetilde{\mathscr{F}}_{n}, L_{2}(Q)\right) \leqslant C \varepsilon^{-V} \quad \forall \varepsilon \in(0,1),
$$

где $C>0$ - константа. Для любого $\varepsilon>0$, любой функции $g \in \widetilde{\mathscr{F}}_{0,1}$ и куба $\mathscr{C} \subset D_{a}$ с ребрами длины $\varepsilon$ мы имеем

$$
\begin{aligned}
K(g, \mathscr{C}) & =\sup _{v} \frac{1}{\|v\|_{\infty}}\left|\int_{\mathscr{C}} g(x) \sum_{i=1}^{d} \frac{\partial v}{\partial x_{i}} d x\right| \leqslant \sup _{v} \frac{1}{\|v\|_{\infty}}\left|\int_{\mathscr{C}} \sum_{i=1}^{d} \frac{\partial g}{\partial x_{i}} v_{i}(x) d x\right| \\
& \leqslant \sup _{v} \frac{1}{\|v\|_{\infty}} h^{d-1} \Psi(s)\left|\int \sum_{i=1}^{d} \frac{\partial K}{\partial z_{i}} v_{i}(s+z h) d z\right| \\
& \leqslant \sup _{v} \Psi(s) h^{d-1} \int\left|\sum_{i=1}^{d} \frac{\partial K(z)}{\partial z_{i}}\right| d z\left(\frac{\varepsilon}{h}\right)^{d} \leqslant \sup _{v} C_{K} \Psi(s) h^{-1} \varepsilon^{d} \\
& \leqslant C_{K} \Psi(s) h^{-1} \varepsilon^{d-1} \varepsilon \leqslant C_{K} \Psi(s) \varepsilon^{d-1} S_{0} \sup _{t \in D_{a}}\left|\Psi(t) f^{\beta}(t)\right| a^{\beta} \varepsilon^{d-1}=K_{\text {loc }}\left(\widetilde{\mathscr{F}}_{0,1}\right) \varepsilon^{d-1} \\
& \leqslant C_{K} S_{0} \sup ^{d}
\end{aligned}
$$

для достаточно больших $n$, где супремум берется по всем бесконечно дифференцируемым функциям $v: \mathscr{C} \rightarrow \mathbf{R}^{d}$ с компактным носителем в $\mathscr{C}$ и $S_{0}$ - максимальная длина ребра для куба, помещающегося внутри носителя $K$ (в третьей строке (5) мы воспользовались условием $\left.\int_{\mathbf{R}^{d}}\left|\sum_{i=1}^{d} \partial K(z) / \partial z_{i}\right| d z \leqslant C_{K}\right)$. Заметим, что $K_{\text {loc }}\left(\widetilde{\mathscr{F}}_{0,1}\right)=$ $C_{K} S_{0}\left\|\Psi f^{\beta}\right\|_{D_{a}} a^{\beta}>1$ для достаточно больших $a$. Таким образом, у $\widetilde{F}_{0,1}$ локально равномерно ограниченная вариация. Более того, условие 
(LUBV) выполнено с константой $K_{\text {loc }}\left(\widetilde{\mathscr{F}}_{0,1}\right)$. Используя рассуждения, подобные (5), можно показать, что

$$
\begin{gathered}
K_{\mathrm{loc}}\left(\widetilde{\mathscr{F}}_{n}\right)=C_{K} S_{0} \sup _{t \in D_{a}}\left|\Psi(t) f^{\beta}(t)\right| a^{\beta} \cdot 2 h_{n}^{-1}, \\
K\left(\widetilde{\mathscr{F}}_{n}\right) \leqslant C_{K} \sup _{t \in D_{a}}\left|\Psi(t) f^{\beta}(t)\right| a^{\beta}\left(2 h_{n}\right)^{d-1} .
\end{gathered}
$$

4. Условно на событии $Z=\left(n_{0}, \ldots, n_{M}\right)$ эмпирические процессы $\widetilde{\nu}_{n_{m}, P_{m}}, m=0, \ldots, M$, независимы и $P_{m}, m=1, \ldots, M$, удовлетворяют лемме 1 . Тогда условно на событии $Z=\left(n_{0}, \ldots, n_{M}\right)$ мы построим независимые броуновские мосты $G_{n_{m}, P_{m}}, m=1, \ldots, M$, индексированные функциями из $\widetilde{\mathscr{F}}_{n}$. Они являются центрированными гауссовскими полями с соответствующими ковариационными функциями

$$
\left(g_{1}, g_{2}\right) \rightarrow \mathbf{E}_{P_{m}}\left(g_{1}\left(Y_{1}^{m}\right) g_{2}\left(Y_{1}^{m}\right)\right)-\mathbf{E}_{P_{m}}\left(g_{1}\left(Y_{1}^{m}\right)\right) \mathbf{E}_{P_{m}}\left(g_{2}\left(Y_{1}^{m}\right)\right),
$$

где $m=1, \ldots, M$. Условно на событии $Z=\left(n_{0}, \ldots, n_{M}\right)$ определим $B_{n_{m}, P_{m}}, m=1, \ldots, M$, - независимые броуновские движения, индексированные функциями из $\widetilde{\mathscr{F}}_{n}$ - из соотношения $G_{n_{m}, P_{m}}=B_{n_{m}, P_{m}}-$ $P_{m} g B_{n_{m}, P_{m}}(1), m=1, \ldots, M$. Они являются центрированными гауссовскими полями с соответствующими ковариационными функциями

$$
\left(g_{1}, g_{2}\right) \rightarrow \mathbf{E}_{P_{m}}\left(g_{1}\left(Y_{1}^{m}\right) g_{2}\left(Y_{1}^{m}\right)\right), \quad m=1, \ldots, M .
$$

При $m=0$ мы не можем применить лемму 1 , поскольку $f$ обращается в нуль на $B_{0}$. Мы определим броуновский мост, индексированный функциями из $\widetilde{\mathscr{F}}_{n}$, как $G_{n_{0}, P_{0}}=0$, тогда формально его ковариационная функция удовлетворяет выражению выше с $m=0$. Он также независим от всех $G_{n_{m}, P_{m}}, m=1, \ldots, M$.

Пусть $\gamma_{m}, m=0, \ldots, M$, - гауссовские случайные величины с нулевыми средними значениями и ковариационной матрицей $\operatorname{Cov}\left(\gamma_{m}, \gamma_{m^{\prime}}\right)=$ $p_{m}\left(I\left(m=m^{\prime}\right)-p_{m^{\prime}}\right), m, m^{\prime}=0, \ldots, M$, независимые от последовательности процессов $G_{n_{m}, P_{m}}$ и $B_{n_{m}, P_{m}}, m=0, \ldots, M$. Затем мы определим броуновский мост, индексированный функциями из $\widetilde{\mathscr{F}}_{n}$, формулой

$$
G_{n, P}=\sum_{m=0}^{M} \sqrt{p_{m}} G_{n_{m}, P_{m}}+\sum_{m=0}^{M} \gamma_{m} P_{m} .
$$

Заметим, что $G_{n, P}$ - центрированное гауссовское поле с ковариационной функцией

$$
\left(g_{1}, g_{2}\right) \rightarrow \mathbf{E}_{P}\left(g_{1}\left(X_{1}\right) g_{2}\left(X_{1}\right)\right)-\mathbf{E}_{P}\left(g_{1}\left(X_{1}\right)\right) \mathbf{E}_{P}\left(g_{2}\left(X_{1}\right)\right) .
$$

Введем также броуновское движение, индексированное функциями из $\widetilde{\mathscr{F}}_{n}$, формулой

$$
B_{n, P}=\sum_{m=0}^{M} \sqrt{p_{m}} B_{n_{m}, P_{m}}
$$


Оно является центрированным гауссовским полем с ковариационной функцией

$$
\left(g_{1}, g_{2}\right) \rightarrow \mathbf{E}_{P}\left(g_{1}\left(X_{1}\right) g_{2}\left(X_{1}\right)\right)
$$

Естественно, имеем $G_{n, P} \stackrel{d}{=} B_{n, P}-P g B_{n, P}(1)$.

Тогда для любой функции $g \in \widetilde{\mathscr{F}}_{n}$

$$
\begin{aligned}
& G_{n, P}(g) \stackrel{d}{=} B_{n, P}(g)-P g B_{n, P}(1) \\
& \quad \stackrel{d}{=} \sum_{m=0}^{M} \sqrt{p_{m}} B_{n_{m}, P_{m}}(g)-\sum_{m=0}^{M} \sqrt{p_{m}} B_{n_{m}, P_{m}}(1) \sum_{m^{\prime}=0}^{M} p_{m^{\prime}} P_{m^{\prime}} g \\
& \quad \stackrel{d}{=} \sum_{m=0}^{M} \sqrt{p_{m}}\left[B_{n_{m}, P_{m}}(g)-P_{m} g B_{n_{m}, P_{m}}(1)\right]+\sum_{m=0}^{M} \sqrt{p_{m}} B_{n_{m}, P_{m}}(1)\left(P_{m} g-P g\right) \\
& \stackrel{d}{=} \sum_{m=0}^{M} \sqrt{p_{m}} G_{n_{m}, P_{m}}+\sum_{m=0}^{M} P_{m} g\left[\sqrt{p_{m}} B_{n_{m}, P_{m}}(1)-p_{m} \sum_{m^{\prime}=0}^{M} \sqrt{p_{m^{\prime}}} B_{n_{m^{\prime}}, P_{m^{\prime}}}(1)\right],
\end{aligned}
$$

где

$$
\begin{aligned}
\operatorname{Cov}( & \sqrt{p_{m}} B_{n_{m}, P_{m}}(1)-p_{m} \sum_{m^{\prime}=0}^{M} \sqrt{p_{m^{\prime}}} B_{n_{m^{\prime}}, P_{m^{\prime}}}(1), \\
& \left.\quad \sqrt{p_{l}} B_{n_{l}, P_{l}}(1)-p_{l} \sum_{l^{\prime}=0}^{M} \sqrt{p_{l^{\prime}}} B_{n_{l^{\prime}}, P_{l^{\prime}}}(1)\right)=p_{m}\left(I(m=l)-p_{l}\right)
\end{aligned}
$$

для $m, l=0, \ldots, M$.

5. При фиксированном $Z=\left(n_{0}, \ldots, n_{M}\right)$ для любых положительных чисел $\tau_{0}, \tau_{1}, \ldots, \tau_{M+2}$ мы имеем

$$
\begin{aligned}
& \mathbf{P}\left(\sup _{g \in \widetilde{\mathscr{F}}_{n}}\left|\sqrt{n} \nu_{n, P}(g)-\sqrt{n} G_{n, P}(g)\right| \geqslant \sum_{m=0}^{M+2} \tau_{m} \mid Z=z\right) \\
& \leqslant \mathbf{P}\left(\sum_{m=0}^{M} \sup _{g \in \widetilde{F}_{n}}\left|\sqrt{n_{m}} \nu_{n_{m}, P_{m}}(g)-\sqrt{n_{m}} G_{n_{m}, P_{m}}(g)\right|\right. \\
& \quad+\sup _{g \in \widetilde{\mathscr{F}}_{n}}\left|\sum_{m=0}^{M}\left[\sqrt{n_{m}}-\sqrt{n p_{m}}\right] G_{n_{m}, P_{m}}(g)\right| \\
& \left.\quad+\sup _{g \in \widetilde{\mathscr{F}}_{n}}\left|\sum_{m=0}^{M}\left[n_{m}-n p_{m}-\sqrt{n} \gamma_{m}\right] P_{m} g\right| \geqslant \sum_{m=0}^{M+2} \tau_{m} \mid Z=z\right) \\
& \leqslant \sum_{m=0}^{M} \mathbf{P}\left(\sup _{g \in \widetilde{\mathscr{F}}_{n}}\left|\sqrt{n_{m}} \nu_{n_{m}, P_{m}}(g)-\sqrt{n_{m}} G_{n_{m}, P_{m}}(g)\right| \geqslant \tau_{m} \mid Z=z\right) \\
& \quad+\mathbf{P}\left(\sup _{g \in \widetilde{\mathscr{F}}_{n}}\left|\sum_{m=0}^{M}\left[\sqrt{n_{m}}-\sqrt{n p_{m}}\right] G_{n_{m}, P_{m}}(g)\right| \geqslant \tau_{M+1} \mid Z=z\right)
\end{aligned}
$$




$$
+\mathbf{P}\left(\sup _{g \in \widetilde{\mathscr{F}}_{n}}\left|\sum_{m=0}^{M}\left[n_{m}-n p_{m}-\sqrt{n} \gamma_{m}\right] P_{m} g\right| \geqslant \tau_{M+2} \mid Z=z\right) .
$$

Далее мы рассмотрим слагаемые в правой части (7) по отдельности.

Используя лемму 1 при заданном $Z=\left(n_{0}, \ldots, n_{M}\right)$ для любого $t_{m} \geqslant$ $C \ln n_{m}$, мы получим

$$
\begin{aligned}
& \mathbf{P}\left(\sup _{g \in \widetilde{F}_{n}}\left|\sqrt{n_{m}} \nu_{n_{m}, P_{m}}(g)-\sqrt{n_{m}} G_{n_{m}, P_{m}}(g)\right|\right. \\
& \left.\quad \geqslant C n_{m}^{(d-1) /(2 d)} h_{n}^{(d-1) / 2} \sqrt{t_{m}}+C t_{m} h_{n}^{-1 / 2} \mid Z=z\right) \leqslant e^{-t_{m}},
\end{aligned}
$$

где $C$ - положительная константа. Мы берем $t_{m}=C_{1} \ln n$ для достаточно большого $C_{1}>0$, что превращает $\tau_{m}=C n^{(d-1) /(2 d)} h_{n}^{(d-1) / 2} \sqrt{\ln n}+$ $C \ln n h_{n}^{-1 / 2}$ для $m=1, \ldots, M$ в (7).

Для процесса $\nu_{n_{0}, P_{0}}$ мы можем применить оценку хвоста $(2.14 .9)$ в [13] для эмпирических процессов, так что

$$
\mathbf{P}\left(\sup _{g \in \widetilde{F}_{n}}\left|\nu_{n_{0}, P_{0}}(g)\right| \geqslant t \mid Z=z\right) \leqslant C t^{V} \exp \left\{-2 t^{2}\right\} \quad \forall t>0,
$$

где $C>0-$ произвольная константа. Положим $t=h_{n}^{d}$. Тогда

$$
\mathbf{P}\left(\sup _{g \in \widetilde{F}_{n}}\left|\nu_{n_{0}, P_{0}}(g)\right| \geqslant \frac{\tau_{0}}{\sqrt{n_{0}}} \mid Z=z\right) \leqslant C h_{n}^{d V} \exp \left\{-2 h_{n}^{2 d}\right\},
$$

где $\tau_{0}=\sqrt{n} h_{n}^{d}$.

6. Оценим последнее слагаемое в правой части (7):

$$
\begin{aligned}
& \mathbf{P}\left(\sup _{g \in \widetilde{\mathscr{F}}_{n}}\left|\sum_{m=0}^{M}\left[n_{m}-n p_{m}-\sqrt{n} \gamma_{m}\right] P_{m} g\right| \geqslant \tau_{M+2} \mid Z=z\right) \\
& \quad \leqslant \mathbf{P}\left(\sup _{m=0, \ldots, M}\left|n_{m}-n p_{m}-\sqrt{n} \gamma_{m}\right| \sum_{m^{\prime}=0}^{M} \sup _{g \in \widetilde{F}_{n}} P_{m^{\prime}} g \geqslant \tau_{M+2} \mid Z=z\right) \\
& \quad \leqslant \mathbf{P}\left(\sup _{m=0, \ldots, M}\left|n_{m}-n p_{m}-\sqrt{n} \gamma_{m}\right| \sum_{m^{\prime}=0}^{M} C p_{m^{\prime}}^{-1} \geqslant \tau_{M+2} \mid Z=z\right) \\
& \quad \leqslant \sum_{m=0}^{M} \mathbf{P}\left(\left|n_{m}-n p_{m}-\sqrt{n} \gamma_{m}\right| \geqslant C_{5} \tau_{M+2} \mid Z=z\right)
\end{aligned}
$$

для некоторых положительных констант $C, C_{5}$.

Пусть $U_{1}, \ldots, U_{n}$ - независимые равномерно распределенные на $[0,1]$ случайные величины, и пусть $W_{n}, n \geqslant 1$, - стандартные броуновские мосты на $[0,1]$. Определим соответствующий эмпирический процесс

$$
\zeta_{n}(t)=n^{-1 / 2} \sum_{i=1}^{n}\left[I\left(U_{i} \in[0, t]\right)-t\right], \quad t \in[0,1] .
$$


Классический результат Я. Комлоша, П. Майора и Г. Тушнади утверждает, что существует версия $W_{n}$, определенная на том же вероятностном пространстве, такая, что для любого $u>0$

$$
\mathbf{P}\left(\sqrt{n} \sup _{t \in[0,1]}\left|\zeta_{n}(t)-W_{n}(t)\right|>u+C_{k m t} \ln n\right) \leqslant L \exp \{-\theta u\},
$$

где $C_{k m t}, L, \theta-$ абсолютные положительные константы. Заметим, что для любого $m=0, \ldots, M$

$$
\sqrt{n} \zeta_{n}\left(\sum_{k=0}^{m} p_{k}\right)-\sqrt{n} \alpha_{n}\left(\sum_{k=0}^{m-1} p_{k}\right) \stackrel{d}{=} n_{m}-n p_{m}
$$

И

$$
W_{n}\left(\sum_{k=0}^{m} p_{k}\right)-W_{n}\left(\sum_{k=0}^{m-1} p_{k}\right) \stackrel{d}{=} \gamma_{m}
$$

где $p_{-1}=0$, т.е. мы можем продолжить цепочку оценок (9) следующим образом

$$
\begin{aligned}
& \sum_{m=0}^{M} \mathbf{P}\left(\left|n_{m}-n p_{m}-\sqrt{n} \gamma_{m}\right| \geqslant C_{5} \tau_{M+2} \mid Z=z\right) \\
& \quad \leqslant \sum_{m=0}^{M} \mathbf{P}\left(2 \sqrt{n} \sup _{t \in[0,1]}\left|\zeta_{n}(t)-W_{n}(t)\right| \geqslant C_{5} \tau_{M+2} \mid Z=z\right) \\
& \quad \leqslant M L \exp \left\{-\frac{\theta C_{5} \tau_{M+2}}{2}\right\} n^{-C_{k m t}}
\end{aligned}
$$

для любого $\tau_{M+2}>2 C_{k m t} \ln n$.

7. Рассмотрим второе слагаемое в правой части (7). Оно может быть ограничено сверху следующим образом:

$$
\begin{aligned}
& \mathbf{P}\left(\sup _{g \in \widetilde{F}_{n}}\left|\sum_{m=0}^{M}\left(\sqrt{n_{m}}-\sqrt{n p_{m}}\right) G_{n_{m}, P_{m}}(g)\right| \geqslant \tau_{M+1} \mid Z=z\right) \\
& \quad \leqslant \mathbf{P}\left(\sup _{m^{\prime}=0, \ldots, M} \sup _{g \in \widetilde{\mathscr{F}}_{n}}\left|G_{n_{m^{\prime}}, P_{m^{\prime}}}(g)\right| \sum_{m=0}^{M}\left|\sqrt{n_{m}}-\sqrt{n p_{m}}\right| \geqslant \tau_{M+1} \mid Z=z\right) .
\end{aligned}
$$

Надо рассмотреть супремум и сумму по отдельности.

Чтобы разобраться с супремумом в (10), мы применяем неравенство Бореля-Цирельсона-Судакова в форме (3.2) в [8] к $G_{n_{m}, P_{m}}, m=0, \ldots, M$, и получаем для любого $t>0$

$$
\mathbf{P}\left(\sup _{g \in \widetilde{\mathscr{F}}_{n}}\left|G_{n_{m}, P_{m}}(g)\right| \geqslant t \mid Z=z\right) \leqslant \exp \left\{-\frac{2\left(t-E_{n_{m}}\right)^{2}}{\pi^{2} \sigma_{n_{m}}^{2}}\right\},
$$


где $\mathbf{E} \sup _{g \in \widetilde{\mathscr{F}}_{n}}\left|G_{n_{m}, P_{m}}(g)\right| \leqslant E_{n_{m}}$ и $\sup _{g \in \widetilde{\mathscr{F}}_{n}} \mathbf{D}\left(G_{n_{m}, P_{m}}(g)\right) \leqslant \sigma_{n_{m}}^{2}$.

Заметим, что для любой функции $g \in \widetilde{\mathscr{F}}_{n}$ и любого $m=0, \ldots, M$

$$
\begin{gathered}
\mathbf{D}\left(G_{n_{m}}(g)\right)=p_{m}^{-1} \int_{B_{m}} g^{2}(t) f(t) d t-\left(p_{m}^{-1} \int_{B_{m}} g(t) f(t) d t\right)^{2} \\
=p_{m}^{-1} \Psi^{2}(x)\left[\int_{\left(x-B_{m}\right) / h} K^{2}(y) f(x-h y) h^{d} d y\right. \\
\left.\quad-p_{m}^{-1}\left(\int_{\left(x-B_{m}\right) / h} K(y) f(x-h y) h^{d} d y\right)^{2}\right] \\
\leqslant p_{m}^{-1} h^{d} \sup _{t \in D_{a}}|\Psi(t)|^{2}\left[\|K\|_{2}^{2}\|f\|_{\infty}+p_{m}^{-1} h^{2 d}\|K\|_{1}^{2}\|f\|_{\infty}^{2}\right] \\
\leqslant 2 p_{m}^{-1} \sup _{t \in D_{a}}|\Psi(t)|^{2}\|K\|_{2}^{2}\|f\|_{\infty} h^{d}
\end{gathered}
$$

для любых $x \in D_{a}$ и $h \in\left[h_{n} / 2,2 h_{n}\right]$. Тогда

$$
\sigma_{m}^{2}=2^{d+1} p_{m}^{-1} \sup _{t \in D_{a}}|\Psi(t)|^{2}\|K\|_{2}^{2}\|f\|_{\infty} h_{n}^{d}=C_{\sigma} p_{m}^{-1} h_{n}^{d} .
$$

Для нахождения $E_{n_{m}}$ мы воспользуемся простым наблюдением

$\mathbf{E} \sup _{g \in \widetilde{\mathscr{F}}_{n}}\left|G_{n_{m}}(g)\right| \leqslant \mathbf{E} \sup _{g \in \widetilde{\mathscr{F}}_{n}}\left|B_{n_{m}}(g)\right|+p_{m}^{-1} \sup _{g \in \widetilde{\mathscr{F}}_{n}}\left|\int_{B_{m}} g(x) f(x) d x\right| \mathbf{E}\left|B_{n_{m}}(1)\right|$ в сочетании с неравенством в духе Дадли

$$
\mathbf{E} \sup _{g \in \widetilde{F}_{n}}\left|B_{n_{m}}(g)\right| \leqslant \mathbf{E}\left|B_{n_{m}}\left(g_{0}\right)\right|+C \int_{0}^{\infty} \sqrt{\ln D\left(\varepsilon, d_{B_{n_{m}}}\right)} d \varepsilon
$$

где $D\left(\varepsilon, d_{B_{n_{m}}}\right)$ - максимальное число $\varepsilon$-разделенных точек по метрике

$$
d_{B_{n_{m}}}\left(g_{1}, g_{2}\right)=\mathbf{D}^{1 / 2}\left(B_{n_{m}}\left(g_{1}\right)-B_{n_{m}}\left(g_{2}\right)\right), \quad g_{1,2} \in \widetilde{\mathscr{F}}_{n}
$$

(см. [13, с. 101]). Поскольку

$$
d_{B_{n_{m}}}^{2}\left(g_{1}, g_{2}\right)=p_{m}^{-1} \int_{B_{m}} f(x)\left(\Psi\left(s_{1}\right) K\left(\frac{s_{1}-x}{h_{1}}\right)-\Psi\left(s_{2}\right) K\left(\frac{s_{2}-x}{h_{2}}\right)\right)^{2} d x
$$

для соответствующих $s_{1,2} \in D_{a}$ и $h_{1,2} \in\left[h_{n} / 2,2 h_{n}\right]$ и выполнены неравенства $N(\varepsilon, d) \leqslant D(\varepsilon, d) \leqslant N(\varepsilon / 2, d)$, мы имеем

$$
D\left(\varepsilon, d_{B_{n_{m}}}\right) \leqslant C \varepsilon^{-V} \quad \forall \varepsilon \in(0,1), \quad D\left(\varepsilon, d_{B_{n_{m}}}\right)=1 \quad \forall \varepsilon>1
$$

и, следовательно,

$$
\begin{aligned}
\int_{0}^{\infty} \sqrt{\ln D\left(\varepsilon, d_{B_{n_{m}}}\right)} d \varepsilon & \leqslant C \int_{0}^{h_{n}^{-1}} \sqrt{\ln C-V \ln \varepsilon} d \varepsilon \\
& \leqslant C \int_{\ln \left(C h_{n}^{V}\right) / V}^{\infty} \sqrt{V t} e^{-t} d t \leqslant C \Gamma\left(\frac{3}{2}\right)<\infty .
\end{aligned}
$$


Таким образом, для любого $m=0, \ldots, M$

$$
\begin{aligned}
\mathbf{E} \sup _{g \in \widetilde{\mathscr{F}}_{n}}\left|B_{n_{m}}(g)\right| & \leqslant \mathbf{E}\left|B_{n_{m}}\left(g_{0}\right)\right|+C_{0} \\
& \leqslant p_{m}^{-1 / 2} \Psi\left(s_{0}\right)\left(\int_{B_{m}} K^{2}\left(\frac{s_{0}-x}{h_{0}}\right) f(x) d x\right)^{1 / 2}+C_{0} \\
& =E<\infty .
\end{aligned}
$$

В результате мы получаем оценку хвоста распределения

$$
\mathbf{P}\left(\sup _{g \in \widetilde{\mathscr{F}}_{n}}\left|G_{n_{m}, P_{m}}(g)\right| \geqslant t \mid Z=z\right) \leqslant \exp \left\{-\frac{2(t-E)^{2} p_{m}}{\pi^{2} C_{\sigma} h_{n}^{d}}\right\}
$$

для любого $t>0$ и любого $m=0, \ldots, M$.

Чтобы разобраться с суммой в (10), мы используем результат шага 6. Сначала заметим, что

$$
\begin{aligned}
\sum_{m=0}^{M}\left|\sqrt{n_{m}}-\sqrt{n p_{m}}\right| \leqslant & M \sup _{m=0, \ldots, M}\left|\sqrt{n_{m}}-\sqrt{n p_{m}}-\sqrt{n} \gamma_{m}\right| \\
& +M \sqrt{n} \sup _{m=0, \ldots, M}\left|\gamma_{m}\right|,
\end{aligned}
$$

так что из шага 6 мы имеем для любых $t_{1}, t_{2}>0$

$$
\begin{aligned}
\mathbf{P}( & \left.\sum_{m=0}^{M}\left|\sqrt{n_{m}}-\sqrt{n p_{m}}\right| \geqslant t_{1}+t_{2} \mid Z=z\right) \\
\leqslant & \mathbf{P}\left(\sup _{m=0, \ldots, M}\left|\sqrt{n_{m}}-\sqrt{n p_{m}}-\sqrt{n} \gamma_{m}\right| \geqslant \frac{t_{1}}{M} \mid Z=z\right) \\
& +\mathbf{P}\left(\sqrt{n} \sup _{m=0, \ldots, M}\left|\gamma_{m}\right| \geqslant \frac{t_{2}}{M} \mid Z=z\right) \\
\leqslant & L \exp \left\{-\frac{\theta t_{1}}{M}\right\} n^{-C_{k m t}} \\
& +\mathbf{P}\left(\sup _{m=0, \ldots, M}\left|W_{n}\left(\sum_{k=0}^{m} p_{k}\right)-W_{n}\left(\sum_{k=0}^{m-1} p_{k}\right)\right| \geqslant n^{-1 / 2} \frac{t_{2}}{M} \mid Z=z\right) \\
\leqslant & L \exp \left\{-\frac{\theta t_{1}}{M}\right\} n^{-C_{k m t}}+\mathbf{P}\left(\sup _{t \in[0,1]}\left|W_{n}(t)\right| \geqslant 2 n^{-1 / 2} \frac{t_{2}}{M} \mid Z=z\right) \\
\leqslant & L \exp \left\{-\frac{\theta t_{1}}{M}\right\} n^{-C_{k m t}}+C \exp \left\{-\frac{8 t_{2}^{2}}{M^{2}}\right\} .
\end{aligned}
$$

Пусть $t_{1}=h_{n}^{d / 2}$ и $t_{2}=C \sqrt{\ln n}$ для некоторого $C>0$. Положим $\tau_{M+1}=$ $\left(h_{n}^{d / 2}+C \sqrt{\ln n}\right)\left(E+\sqrt{h_{n}^{d} \ln n}\right)$, тогда

$$
\mathbf{P}\left(\sup _{m^{\prime}=0, \ldots, M} \sup _{g \in \widetilde{\mathscr{F}}_{n}}\left|G_{n_{m^{\prime}}, P_{m^{\prime}}}(g)\right| \sum_{m=0}^{M}\left|\sqrt{n_{m}}-\sqrt{n p_{m}}\right| \geqslant \tau_{M+1} \mid Z=z\right)
$$




$$
\begin{aligned}
\leqslant & \mathbf{P}\left(\sum_{m=0}^{M}\left|\sqrt{n_{m}}-\sqrt{n p_{m}}\right| \geqslant h_{n}^{d / 2}+C \sqrt{\ln n} \mid Z=z\right) \\
& +\mathbf{P}\left(\sup _{m^{\prime}=0, \ldots, M} \sup _{g \in \widetilde{F}_{n}}\left|G_{n_{m^{\prime}}, P_{m^{\prime}}}(g)\right| \geqslant E+\sqrt{h_{n}^{d} \ln n} \mid Z=z\right) \\
\leqslant & L \exp \left\{-\frac{\theta h_{n}^{d / 2}}{M}\right\} n^{-C_{k m t}}+C n^{-8 C^{2} / M^{2}}+n^{-C}
\end{aligned}
$$

для некоторой положительной конечной константы $C$.

8. Складывая все части воедино и полагая $\tau_{0}=\sqrt{n} h_{n}^{d}$,

$$
\begin{gathered}
\tau_{m}=C n^{(d-1) /(2 d)} h_{n}^{(d-1) / 2} \sqrt{\ln n}+C h_{n}^{-1 / 2} \ln n, \quad m=1, \ldots, M, \\
\tau_{M+1}=\left(h_{n}^{d / 2}+\sqrt{\ln n}\right)\left(E+C h_{n}^{d / 2} \sqrt{\ln n}\right), \quad \tau_{M+2}=3 C_{k m t} \ln n
\end{gathered}
$$

мы получаем

$$
\begin{aligned}
& \mathbf{P}\left(\sup _{g \in \widetilde{\mathscr{F}}_{n}}\left|\sqrt{n} \nu_{n, P}(g)-\sqrt{n} G_{n, P}(g)\right| \geqslant \sum_{m=0}^{M+2}\right) \\
& \leqslant C h_{n}^{d V} \exp \left\{-2 h_{n}^{2 d}\right\}+M n^{-C_{1}}+n^{-C}+L \exp \left\{-\frac{\theta h_{n}^{C}}{M}\right\} n^{-C_{k m t}} \\
& \quad+n^{-\left(1+1.5 \theta C_{5}\right) C_{k m t}} \leqslant n^{-C_{8}}
\end{aligned}
$$

для некоторого достаточно большого $C_{8}>0$, поскольку $n^{-\eta_{1} / d} \leqslant h_{n} \leqslant$ $n^{-\eta_{0} / d}$ из условия (Н2). Отсюда вытекает

$$
\begin{aligned}
& \mathbf{P}\left(\alpha_{n} h_{n}^{-d / 2} \sup _{g \in \widetilde{\mathscr{F}}_{n}}\left|\nu_{n, P}(g)-G_{n, P}(g)\right| \geqslant \alpha_{n} h_{n}^{d / 2}+M C \alpha_{n} h_{n}^{-1 / 2} n^{-1 /(2 d)} \sqrt{\ln n}\right. \\
& \quad+M C \alpha_{n} h_{n}^{-(d+1) / 2} n^{-1 / 2} \ln n+E \alpha_{n} n^{-1 / 2}+E \alpha_{n} h_{n}^{-d / 2} n^{-1 / 2} \sqrt{\ln n} \\
& \left.\quad+C \alpha_{n} h_{n}^{d / 2} n^{-1 / 2} \sqrt{\ln n}+C \alpha_{n} n^{-1 / 2} \ln n+3 C_{k m t} \alpha_{n} h_{n}^{-d / 2} n^{-1 / 2} \ln n\right) \leqslant n^{-C_{8}} .
\end{aligned}
$$

Далее следует, что последовательности

$$
\alpha_{n}\left(h_{n}^{-d / 2}\left\|\nu_{n, P}\right\|_{\widetilde{F}_{n}}-\alpha_{n}\right) \quad \text { и } \quad \alpha_{n}\left(h_{n}^{-d / 2}\left\|G_{n, P}\right\|_{\widetilde{F}_{n}}-\alpha_{n}\right)
$$

при $n \rightarrow \infty$ сходятся или расходятся по распределению одновременно, если выполнены следующие соотношения:

$$
\begin{gathered}
\alpha_{n}=o\left(h_{n}^{-d / 2}\right), \quad \alpha_{n}=o\left(\sqrt{n^{1 / d} h_{n} \ln n}\right), \quad \alpha_{n}=o\left(\sqrt{n h_{n}^{d+1}} \ln n\right) \\
\alpha_{n}=o\left(\frac{\sqrt{n}}{\ln n}\right), \quad \alpha_{n}=o\left(\frac{\sqrt{n h_{n}^{d}}}{\ln n}\right), \quad \alpha_{n}=o\left(\sqrt{\frac{n}{h_{n}^{d / 2} \ln n}}\right)
\end{gathered}
$$

9. Оставшаяся часть доказательства похожа на рассуждения в [4], где можно найти подробности. Мы имеем

$$
\mathbf{E}\left\|\alpha_{n} h_{n}^{-d / 2}\left(G_{n, P}-B_{n, P}\right)\right\|_{\mathscr{F}_{n}} \leqslant \alpha_{n} h_{n}^{d / 2}\|K\|_{1}\|f\|_{\infty} \sup _{t \in D_{a}}|\Psi(t)|,
$$


откуда вытекает, что если $\alpha_{n}=o\left(h_{n}^{-d / 2}\right)$, то последовательности

$$
\alpha_{n}\left(h_{n}^{-d / 2}\left\|G_{n, P}\right\|_{\mathscr{F}_{n}}-\alpha_{n}\right) \quad \text { и } \quad \alpha_{n}\left(h_{n}^{-d / 2}\left\|B_{n, P}\right\|_{\mathscr{F}_{n}}-\alpha_{n}\right)
$$

при $n \rightarrow \infty$ сходятся или расходятся по распределению одновременно.

10. Для любого $x \in D_{a}$ определим процесс

$$
Y_{n}\left(\Psi(x) \sqrt{f(x)} K\left(\frac{x-\cdot}{h_{n}}\right)=h_{n}^{-d / 2} \Psi(x) \sqrt{f(x)} \int_{\mathbf{R}^{d}} K\left(\frac{x-s}{h_{n}}\right) d B(s),\right.
$$

где $B$ - это стандартное броуновское движение на $\mathbf{R}^{d}$. Заметим, что для любого $x \in D_{a}$

$$
h_{n}^{-d / 2} B_{n, P}\left(\Psi(x) K\left(\frac{x-\cdot}{h_{n}}\right)=h_{n}^{-d / 2} \Psi(x) \int_{\mathbf{R}^{d}} K\left(\frac{x-s}{h_{n}}\right) \sqrt{f(s)} d B(s) .\right.
$$

Применяя неравенство Бореля-Цирельсона-Судакова и энтропийную оценку Дадли для различных функциональных классов Вапника-Червоненкиса, нетрудно показать, что если $\alpha_{n}=o\left(h_{n}^{-\gamma / 2}\left|\ln h_{n}\right|^{-1 / 2}\right)$, то последовательности

$$
\alpha_{n}\left(h_{n}^{-d / 2}\left\|B_{n, P}\right\|_{\mathscr{F}_{n}}-\alpha_{n}\right) \quad \text { и } \quad \alpha_{n}\left(\left\|Y_{n}\right\|_{\mathscr{F}_{n}}-\alpha_{n}\right)
$$

при $n \rightarrow \infty$ сходятся или расходятся по распределению одновременно. Напомним, что $\gamma$ - показатель из условия Гёльдера функции $f$, введенный в предположении (F1). См. подробности в [4].

Наконец, легко видеть, что для любого $x \in D_{a}$

$$
Y_{n}\left(\Psi(x) \sqrt{f(x)} K\left(\frac{x-\cdot}{h_{n}}\right) \stackrel{d}{=} \Psi(x) \sqrt{f(x)} X\left(\frac{x}{h_{n}}\right)\right.
$$

где $X(y), y \in \mathbf{R}^{d}$, — центрированное гауссовское поле с ковариационной функцией

$$
r(y)=\int_{\mathbf{R}^{d}} K(y+s) K(s) d s, \quad y \in \mathbf{R}^{d} .
$$

И поскольку $\sup _{g \in \mathscr{F}_{n}}\left|Y_{n}(g)\right|=\sup _{y \in D_{a}}\left|\Psi(y) \sqrt{f(y)} X\left(y / h_{n}\right)\right|$, то доказательство теоремы 4 завершено.

4. Доказательство теоремы 1. Данная теорема обобщает результаты В.И. Питербарга [11] для супремумов стационарных гауссовских полей на компактных множествах до всего пространства $\mathbf{R}^{d}$. Специальным образом выбранная весовая функция необходима, чтобы обеспечить сходимость. В доказательстве мы собираемся эксплуатировать результат для компактных множеств, который мы формулируем ниже для полноты. Но сначала введем некоторые дополнительные обозначения. 
Пусть $k \in \mathbf{N}$ и пусть $l=\left(l_{1}, \ldots, l_{k+1}\right)$ и $\tau=\left(\tau_{1}, \ldots, \tau_{k}\right)$ - мультииндексы такие, что $1=l_{1}<\cdots<l_{k+1}=d+1$ и $\tau_{1}, \ldots, \tau_{k} \in(0,2]$. Для $x \in \mathbf{R}^{d}$ обозначим

$$
\|x\|_{\tau}:=\sum_{i=1}^{k}\left(\sum_{j=l_{i}}^{l_{i+1}-1} x_{j}^{2}\right)^{\tau_{i} / 2}
$$

Пусть $\varphi(u), u \in \mathbf{R}$, - плотность стандартной нормальной величины.

Следующее утверждение - это лемма 7.1 в сочетании со следствием 7.3 в [5]. См. также теорему 8 в [9, гл. 14].

Лемма 2. Пусть ковариационная функиия $\rho(x), x \in \mathbf{R}^{d}$, иентрированного гауссовского однородного поля $Y(x), x \in \mathbf{R}^{d}$, ведет себя около 0 как

$$
\rho(x)=1-\|x\|_{\tau}+o\left(\|x\|_{\tau}\right), \quad\|x\|_{\tau} \rightarrow 0,
$$

для некоторьх $k, \tau, l$. Также предположим, ито $\sup _{\|x\|>\varepsilon}|\rho(x)|<1$ для всех $\varepsilon>0$. Тогда существует $\delta_{0}>0$ такое, что для любого замкнутого измеримого по Жордану множества $S$ ненулевой меры с диаметром, не превьиающим $\delta_{0}$, мьи имеем при $u \rightarrow \infty$

$$
\mathbf{P}\left\{\sup _{x \in S}|Y(x)|>u\right\}=2 H_{\tau, l} \text { meas }(S) u^{2 \sum_{i=1}^{k}\left(l_{i+1}-l_{i}\right) / \tau_{i}-1} \varphi(u)(1+o(1)),
$$

где $H_{\tau, l}>0$ - это константа, зависящая только от $\tau$ ul.

Заметим, что для $k=1$ мы имеем $l=(1, d+1),\|x\|_{\tau}=\|x\|^{\tau}$ и $H_{\tau, l}=$ $H_{\tau}$ - константа Пикандса, определенная в разделе 1 . Мы воспользуемся леммой 2 для случая $k=1$ на этапах 4 и 5 и для случая $k=2$ на этапе 6 .

Доказательство теоремы 1 весьма техническое и замысловатое, поэтому мы разделили его на несколько этапов для лучшего изложения.

1. Для фиксированного $t \in \mathbf{R}$ обозначим $u_{T}=t / A_{T}+A_{T}$. Тогда при $T \rightarrow \infty$

$$
T^{d} \Lambda_{d, \alpha}\left(u_{T}\right) \rightarrow e^{-t}
$$

Доказательство почти идентично шагу 1 в [4].

2. Мы можем заменить функции $w(s / T), s \in \mathbf{R}^{d}$, в теореме 1 на следующие:

$$
\begin{aligned}
w_{T}(s):= & w\left(\frac{k}{T}\right) \quad \text { для } k_{i} \leqslant s_{i}<k_{i}+1, k_{i} \in \mathbf{Z}, i=1, \ldots, d, \\
& {\left[\frac{k_{1}}{T}, \frac{k_{1}+1}{T}\right) \times \cdots \times\left[\frac{k_{d}}{T}, \frac{k_{d}+1}{T}\right) \subset W, }
\end{aligned}
$$

в противном случае $w_{T}(s)=0$. Доказательство почти идентично шагу 2 B [4]. 
3. Определим

$$
P_{0}=\mathbf{P}\left\{\sup _{s \in \mathbf{R}^{d}} w_{T}(s)|X(s)|>u_{T}\right\}
$$

Пусть $h>0$, обозначим

$$
S_{1}=\bigcup_{m \in \mathbf{Z}^{d}}\left\{(h+1) m+[0, h]^{d}\right\}=\bigcup_{m \in \mathbf{Z}^{d}} \Delta_{m}(h), \quad S_{2}=\mathbf{R}^{d} \backslash S_{1} .
$$

Затем определим

$$
P_{i}=\mathbf{P}\left\{\sup _{s \in S_{i}} w_{T}(s)|X(s)|>u_{T}\right\}, \quad i=1,2 .
$$

Очевидно, что

$$
\begin{gathered}
P_{1} \leqslant P_{0} \leqslant P_{1}+P_{2}, \\
\ln \left(1-P_{1}\right)-\frac{P_{2}}{1-P_{1}-P_{2}} \leqslant \ln \left(1-P_{1}-P_{2}\right) \leqslant \ln \left(1-P_{0}\right) \leqslant \ln \left(1-P_{1}\right) \leqslant-P_{1} .
\end{gathered}
$$

4. Мы покажем, что для положительной константы $C$ мы имеем при $T \rightarrow \infty$

$$
P_{2} \leqslant C e^{-t} h^{-1}\left(1+o_{T}(1)\right)
$$

Определим

$$
L_{k}(h)=\left((h+1) k+[0, h+1]^{d}-[0, h]^{d}\right) \cap W, \quad k \in \mathbf{Z}^{d} .
$$

Тогда $S_{2}=\bigcup_{k \in \mathbf{Z}^{d}} L_{k}(h)$ и мы подразбиваем каждое $L_{k}(h)$ на маленькие кубики со стороной $\delta$ такие, что их диаметры не превышают $\delta_{0}$ из леммы 2. Точнее, для каждого $[0, h+1]^{d}$-куба мы разделим каждую сторону длины $h+1$ на $(h+1) / \delta$ подынтервалов таким образом, что соответствующая сторона длины $h$ у $[0, h]^{d}$-подкубика состоит из целого числа подынтервалов длины $\delta$. Затем мы берем только те $\delta^{d}$-кубики, которые лежат внутри $L_{k}(h)$. Обозначим их $Q_{k, m}(h, \delta)$; таким образом,

$$
L_{k}(h)=\bigcup_{m \in M_{k}(h, \delta)} Q_{k, m}(h, \delta), \quad Q_{k, m_{1}}(h, \delta) \cap Q_{k, m_{2}}(h, \delta)=\varnothing, \quad m_{1} \neq m_{2} .
$$

Теперь мы имеем

$$
\begin{aligned}
P_{2} & \leqslant \sum_{k \in \mathbf{Z}^{d}} \sum_{m \in M_{k}(h, \delta)} \mathbf{P}\left\{\sup _{s \in Q_{k, m}(h, \delta)} w_{T}(s)|X(s)|>u_{T}\right\} \\
& \leqslant \sum_{k \in \mathbf{Z}^{d}} \sum_{m \in M_{k}(h, \delta)} \mathbf{P}\left\{\sup _{s \in Q_{k, m}(h, \delta)}|X(s)|>\frac{u_{T}}{w\left(m_{k}^{(h, \delta)} / T\right)}\right\},
\end{aligned}
$$


где

$$
w\left(\frac{m_{k}^{(h, \delta)}}{T}\right)=\max _{l \in \mathbf{Z}^{d} \cap Q_{k, m}(h, \delta)} w\left(\frac{l}{T}\right)
$$

положительно, поскольку мы работаем на носителе $W$ функции $w$. По лемме 2 для любого $\varepsilon>0$ и для всех достаточно больших $h$ выражение в (12) допускает оценку

$$
\begin{aligned}
& P_{2} \leqslant(1+\varepsilon) \sum_{k \in \mathbf{Z}^{d}} \sum_{m \in M_{k}(h, \delta)} \operatorname{meas}\left(Q_{k, m}(h, \delta)\right) \\
& \times 2 H_{\alpha}\left(\frac{u_{T}}{w\left(m_{k}^{(h, \delta)} / T\right)}\right)^{2 d / \alpha-1} \varphi\left(\frac{u_{T}}{w\left(m_{k}^{(h, \delta)} / T\right)}\right) \\
& =(1+\varepsilon) \delta^{d} \sum_{k \in \mathbf{Z}^{d}} \sum_{m \in M_{k}(h, \delta)} \Phi_{d, \alpha}\left(\frac{u_{T}}{w\left(m_{k}^{(h, \delta)} / T\right)}\right) \\
& \leqslant C(1+\varepsilon) \int_{S_{2}} \Phi_{d, \alpha}\left(\frac{u_{T}}{w(y / T)}\right) d y \\
& \stackrel{(a)}{\leqslant} C(1+\varepsilon) h^{-1} \int_{\mathbf{R}^{d}} \Phi_{d, \alpha}\left(\frac{u_{T}}{w(y / T)}\right) d y \stackrel{(b)}{\leqslant} C(1+\varepsilon) h^{-1} e^{-x}\left(1+o_{T}(1)\right) \text {, }
\end{aligned}
$$

где $C$ - положительная константа. Мы воспользовались условиями (w1) и $($ w3) в $\stackrel{(a)}{\leqslant}$ и результатом этапа 1 в $\stackrel{(b)}{\leqslant}$.

5. Покажем, что $P_{1} \leqslant e^{-t}\left(1+o_{T}(1)\right)$ при $T \rightarrow \infty$.

Подобно доказательству этапа 4 мы разделим $S_{1}$ на маленькие кубики со стороной $\delta$ такие, что их диаметры не превышают $\delta_{0}$ из леммы 2. Точнее,

$$
\begin{gathered}
S_{1}=\bigcup_{k \in \mathbf{Z}^{d}} \Delta_{k}(h)=\bigcup_{k \in \mathbf{Z}^{d}} \bigcup_{m \in M_{k}(h, \delta)} Q_{k, m}(h, \delta), \\
Q_{k, m_{1}}(h, \delta) \cap Q_{k, m_{2}}(h, \delta)=\varnothing, \quad m_{1} \neq m_{2} .
\end{gathered}
$$

Тогда

$$
\begin{aligned}
P_{1} & \leqslant \sum_{k \in \mathbf{Z}^{d}} \sum_{m \in M_{k}(h, \delta)} \mathbf{P}\left\{\sup _{s \in Q_{k, m}(h, \delta)} w_{T}(s)|X(s)|>u_{T}\right\} \\
& \leqslant \sum_{k \in \mathbf{Z}^{d}} \sum_{m \in M_{k}(h, \delta)} \mathbf{P}\left\{\sup _{s \in Q_{k, m}(h, \delta)}|X(s)|>\frac{u_{T}}{w\left(m_{k}^{(h, \delta)} / T\right)}\right\} \\
& \leqslant(1+\varepsilon) \sum_{k \in \mathbf{Z}^{d}} \sum_{m \in M_{k}(h, \delta)} \operatorname{meas}\left(Q_{k, m}(h, \delta)\right) \Phi_{d, \alpha}\left(\frac{u_{T}}{w\left(m_{k}^{(h, \delta)} / T\right)}\right) \\
& \leqslant(1+\varepsilon) \int_{\mathbf{R}^{d}} \Phi_{d, \alpha}\left(\frac{u_{T}}{w(y / T)}\right) d y=(1+\varepsilon) T^{d} \int_{\mathbf{R}^{d}} \Phi_{d, \alpha}\left(\frac{u_{T}}{w(z)}\right) d z \\
& =(1+\varepsilon) e^{-t}\left(1+o_{T}(1)\right),
\end{aligned}
$$

где $w\left(m_{k}^{(h, \delta)} / T\right)=\max _{l \in \mathbf{Z}^{d} \cap Q_{k, m}(h, \delta)} w(l / T)$. 
6. Покажем, что при $T \rightarrow \infty$

$$
P_{1} \geqslant e^{-t}\left(1+o_{T}(1)\right)
$$

Используя неравенство двойной суммы, мы имеем

$$
\begin{aligned}
P_{1}= & \mathbf{P}\left\{\bigcup_{k \in \mathbf{Z}^{d}}\left\{\sup _{s \in L_{k}(h)} w_{T}(s)|X(s)|>u_{T}\right\}\right\} \\
\geqslant & \sum_{k \in \mathbf{Z}^{d}} \mathbf{P}\left\{\sup _{s \in L_{k}(h)} w_{T}(s)|X(s)|>u_{T}\right\} \\
& -\sum \sum_{k \neq m \in \mathbf{Z}^{d}} \mathbf{P}\left\{\sup _{s \in L_{k}(h)} w_{T}(s)|X(s)|>u_{T}, \sup _{s \in L_{m}(h)} w_{T}(s)|X(s)|>u_{T}\right\} .
\end{aligned}
$$

6а. Покажем, что при $T \rightarrow \infty$

$$
\begin{gathered}
\sum \sum_{k \neq m \in \mathbf{Z}^{d}} \mathbf{P}\left\{\sup _{s \in L_{k}(h)} w_{T}(s)|X(s)|>u_{T}, \sup _{s \in L_{m}(h)} w_{T}(s)|X(s)|>u_{T}\right\} \\
\leqslant e^{-t} o_{T}(1) .
\end{gathered}
$$

Чтобы разобраться с двойной суммой, мы будем следовать доказательствам леммы 6.3 и общей версии леммы 7.1 в [11]. Используя обозначения из предыдущих шагов для $w\left(m_{k}^{(h, \delta)} / T\right)=\max _{l \in \mathbf{Z}^{d} \cap Q_{k, m}(h, \delta)} w(l / T)$, двойную сумму можно ограничить сверху следующим образом:

$$
\begin{aligned}
& \sum \sum_{k \neq m \in \mathbf{Z}^{d}} \mathbf{P}\left\{\sup _{s \in L_{k}(h)} w_{T}(s)|X(s)|>u_{T}, \sup _{s \in L_{m}(h)} w_{T}(s)|X(s)|>u_{T}\right\} \\
& \leqslant \sum_{k \in \mathbf{Z}^{d}} \sum_{i \in M_{k}(h, \delta)} \sum_{m \in \mathbf{Z}^{d}} \sum_{j \in M_{m}(h, \delta)} \mathbf{P}\left\{\sup _{s \in Q_{k, i}(h, \delta)}|X(s)|>\frac{u_{T}}{w\left(i_{k}^{(h, \delta)} / T\right)},\right. \\
& \left.\sup _{s \in Q_{m, j}(h, \delta)}|X(s)|>\frac{u_{T}}{w\left(j_{m}^{(h, \delta)} / T\right)}\right\} \\
& \leqslant \sum_{k \in \mathbf{Z}^{d}} \sum_{i \in M_{k}(h, \delta)} \sum_{m \in \mathbf{Z}^{d}} \sum_{j \in M_{m}(h, \delta)} 2 \mathbf{P}\left\{\sup _{\left(s_{1}, s_{2}\right) \in Q_{k, i}(h, \delta) \times Q_{m, j}(h, \delta)} Y\left(s_{1}, s_{2}\right)\right. \\
& \left.>\frac{u_{T}}{w\left(i_{k}^{(h, \delta)} / T\right)}+\frac{u_{T}}{w\left(j_{m}^{(h, \delta)} / T\right)}\right\},
\end{aligned}
$$

где $Y\left(s_{1}, s_{2}\right)=X\left(s_{1}\right)+X\left(s_{2}\right),\left(s_{1}, s_{2}\right) \in \mathbf{R}^{2 d}$, - гауссовское поле с дисперсией

$$
\sigma_{Y}^{2}\left(s_{1}, s_{2}\right)=2+2 r\left(s_{1}-s_{2}\right),
$$

которая равна 2 на $Q_{k, i}(h, \delta) \times Q_{m, j}(h, \delta)$ в силу условия $(\mathrm{R} 2)$. Последнее неравенство вытекает из того, что $X(\cdot)$ обладает тем же распределением, что и $-X(\cdot)$ всюду в $\mathbf{R}^{d}$. 
Рассмотрим нормированное поле

$$
Y^{*}\left(s_{1}, s_{2}\right)=\frac{Y\left(s_{1}, s_{2}\right)}{\sigma_{Y}\left(s_{1}, s_{2}\right)}=\frac{Y\left(s_{1}, s_{2}\right)}{\sqrt{2}}, \quad\left(s_{1}, s_{2}\right) \in Q_{k, i}(h, \delta) \times Q_{m, j}(h, \delta) .
$$

Мы видим, что при любых $\left(s_{1}, s_{2}\right),\left(s_{1}^{\prime}, s_{2}^{\prime}\right) \in Q_{k, i}(h, \delta) \times Q_{m, j}(h, \delta)$ имеют место соотношения

$$
\begin{aligned}
\mathbf{E}\left(Y^{*}\left(s_{1}, s_{2}\right) Y^{*}\left(s_{1}^{\prime}, s_{2}^{\prime}\right)\right)= & \frac{1}{2}\left(r\left(s_{1}-s_{1}^{\prime}\right)+r\left(s_{1}-s_{2}^{\prime}\right)+r\left(s_{1}^{\prime}-s_{2}\right)+r\left(s_{2}-s_{2}^{\prime}\right)\right) \\
= & \frac{1}{2}\left(r\left(s_{1}-s_{1}^{\prime}\right)+r\left(s_{2}-s_{2}^{\prime}\right)\right) \\
\geqslant & 1-2\left\|s_{1}-s_{1}^{\prime}\right\|^{\alpha}+4\left\|s_{1}-s_{1}^{\prime}\right\|^{2 \alpha} \\
& -2\left\|s_{2}-s_{2}^{\prime}\right\|^{\alpha}+4\left\|s_{2}-s_{2}^{\prime}\right\|^{2 \alpha}
\end{aligned}
$$

для всех достаточно малых $\delta>0$.

А теперь пусть $Z_{1}(s), Z_{2}(s), s \in \mathbf{R}^{d}$, - два независимых центрированных однородных гауссовских поля с ковариационными функциями $r_{Z}\left(s, s_{1}\right)=\exp \left\{-4\left\|s-s_{1}\right\|^{\alpha}\right\}$. Тогда однородное гауссовское поле

$$
Z^{*}\left(s_{1}, s_{2}\right)=\frac{Z_{1}\left(s_{1}\right)+Z_{2}\left(s_{2}\right)}{\sqrt{2}}, \quad\left(s_{1}, s_{2}\right) \in Q_{k, i}(h, \delta) \times Q_{m, j}(h, \delta),
$$

имеет ковариацию

$$
\begin{aligned}
& \frac{1}{2}\left(\exp \left\{-\left\|s_{1}-s_{1}^{\prime}\right\|^{\alpha}\right\}+\exp \left\{-\left\|s_{2}-s_{2}^{\prime}\right\|^{\alpha}\right\}\right) \\
& \quad \leqslant 1-2\left\|s_{1}-s_{1}^{\prime}\right\|^{\alpha}+4\left\|s_{1}-s_{1}^{\prime}\right\|^{2 \alpha}-2\left\|s_{2}-s_{2}^{\prime}\right\|^{\alpha}+4\left\|s_{2}-s_{2}^{\prime}\right\|^{2 \alpha} \\
& \quad \leqslant \mathbf{E}\left(Y^{*}\left(s_{1}, s_{2}\right) Y^{*}\left(s_{1}^{\prime}, s_{2}^{\prime}\right)\right)
\end{aligned}
$$

для всех $\left(s_{1}, s_{2}\right),\left(s_{1}^{\prime}, s_{2}^{\prime}\right) \in Q_{k, i}(h, \delta) \times Q_{m, j}(h, \delta)$, поскольку $e^{-u} \leqslant 1-$ $u+u^{2} / 2$ для любого $u \geqslant 0$. Тогда из неравенства Слепяна (см. [11, теорема С.1]) следует, что

$$
\begin{aligned}
& \mathbf{P}\left\{\sup _{\left(s_{1}, s_{2}\right) \in Q_{k, i}(h, \delta) \times Q_{m, j}(h, \delta)} Y\left(s_{1}, s_{2}\right)>\frac{u_{T}}{w\left(i_{k}^{(h, \delta)} / T\right)}+\frac{u_{T}}{w\left(j_{m}^{(h, \delta)} / T\right)}\right\} \\
& \leqslant \mathbf{P}\left\{\sup _{\left(s_{1}, s_{2}\right) \in Q_{k, i}(h, \delta) \times Q_{m, j}(h, \delta)} Z^{*}\left(s_{1}, s_{2}\right)>\frac{1}{\sqrt{2}}\left(\frac{u_{T}}{w\left(i_{k}^{(h, \delta)} / T\right)}+\frac{u_{T}}{w\left(j_{m}^{(h, \delta)} / T\right)}\right)\right\} .
\end{aligned}
$$

Теперь мы можем применить лемму 2 с $k=2, \tau=(\alpha, \alpha)$, чтобы получить

$$
\begin{aligned}
& \mathbf{P}\left\{\sup _{\left(s_{1}, s_{2}\right) \in Q_{k, i}(h, \delta) \times Q_{m, j}(h, \delta)} Z^{*}\left(s_{1}, s_{2}\right)>\frac{1}{\sqrt{2}}\left(\frac{u_{T}}{w\left(i_{k}^{(h, \delta)} / T\right)}+\frac{u_{T}}{w\left(j_{m}^{(h, \delta)} / T\right)}\right)\right\} \\
& \leqslant(1+\varepsilon) \cdot 2^{2 d / \alpha} \operatorname{meas}\left(Q_{k, i}(h, \delta) \times Q_{m, j}(h, \delta)\right) \\
& \quad \times H_{\alpha, \alpha}\left(\frac{1}{\sqrt{2}}\left(\frac{u_{T}}{w\left(i_{k}^{(h, \delta)} / T\right)}+\frac{u_{T}}{w\left(j_{m}^{(h, \delta)} / T\right)}\right)\right)^{4 d / \alpha-1} \\
& \quad \times \varphi\left(\frac{1}{\sqrt{2}}\left(\frac{u_{T}}{w\left(i_{k}^{(h, \delta)} / T\right)}+\frac{u_{T}}{w\left(j_{m}^{(h, \delta)} / T\right)}\right)\right)
\end{aligned}
$$


и

$$
\begin{aligned}
& \sum \sum_{k \neq m \in \mathbf{Z}^{d}} \mathbf{P}\left\{\sup _{s \in L_{k}(h)} w_{T}(s)|X(s)|>u_{T}, \sup _{s \in L_{m}(h)} w_{T}(s)|X(s)|>u_{T}\right\} \\
& \leqslant \sum_{k \in \mathbf{Z}^{d}} \sum_{i \in M_{k}(h, \delta)} \sum_{m \in \mathbf{Z}^{d}} \sum_{j \in M_{m}(h, \delta)} 2(1+\varepsilon) 2^{2 d / \alpha} \delta^{2 d} \\
& \quad \times H_{\alpha, \alpha}\left(\frac{1}{\sqrt{2}}\left(\frac{u_{T}}{w\left(i_{k}^{(h, \delta)} / T\right)}+\frac{u_{T}}{w\left(j_{m}^{(h, \delta)} / T\right)}\right)\right)^{4 d / \alpha-1} \\
& \quad \times \varphi\left(\frac{1}{\sqrt{2}}\left(\frac{u_{T}}{w\left(i_{k}^{(h, \delta)} / T\right)}+\frac{u_{T}}{w\left(j_{m}^{(h, \delta)} / T\right)}\right)\right)
\end{aligned}
$$

где $H_{\alpha, \alpha}=\lim _{M \rightarrow \infty} M^{-2 d} \mathbf{E} \exp \left\{\max _{\left(s_{1}, s_{2}\right) \in[0, M]^{2 d}} \chi\left(s_{1}, s_{2}\right)\right\}$ и $\chi\left(s_{1}, s_{2}\right)$, $(t, s) \in \mathbf{R}^{2 d}$, - гауссовское поле с непрерывными траекториями, функцией математического ожидания $\mathbf{E} \chi\left(s_{1}, s_{2}\right)=-4\left\|s_{1}\right\|^{\alpha}-4\left\|s_{2}\right\|^{\alpha}$ и ковариационной функцией $r_{\chi}\left(s_{1}, s_{2}, s_{1}^{\prime}, s_{2}^{\prime}\right)=4\left\|s_{1}\right\|^{\alpha}+4\left\|s_{2}\right\|^{\alpha}+4\left\|s_{1}^{\prime}\right\|^{\alpha}+4\left\|s_{2}^{\prime}\right\|^{\alpha}-$ $4\left\|s_{1}-s_{1}^{\prime}\right\|^{\alpha}-4\left\|s_{2}-s_{2}^{\prime}\right\|^{\alpha}$.

Более того, рассуждая аналогично этапам 4 и 5 , получаем оценку

$$
\begin{aligned}
& \sum \sum_{k \neq m \in \mathbf{Z}^{d}} \mathbf{P}\left\{\sup _{s \in L_{k}(h)} w_{T}(s)|X(s)|>u_{T}, \sup _{s \in L_{m}(h)} w_{T}(s)|X(s)|>u_{T}\right\} \\
& \leqslant \frac{(1+\varepsilon) H_{\alpha, \alpha}}{\sqrt{2 \pi}} \cdot 2^{2 d / \alpha+1} \int_{S_{1}} \int_{S_{1}}\left(\frac{1}{\sqrt{2}}\left(\frac{u_{T}}{w\left(y_{1} / T\right)}+\frac{u_{T}}{w\left(y_{2} / T\right)}\right)\right)^{4 d / \alpha-1} \\
& \quad \times \exp \left\{-\frac{1}{4}\left(\frac{u_{T}}{w\left(y_{1} / T\right)}+\frac{u_{T}}{w\left(y_{2} / T\right)}\right)^{2}\right\} d y_{1} d y_{2} \\
& \leqslant \frac{(1+\varepsilon) H_{\alpha, \alpha}}{\sqrt{2 \pi}} \cdot 2^{5 / 2} T^{2 d} \int_{\mathbf{R}^{d}}\left(\frac{u_{T}}{w\left(z_{1}\right)}\right)^{2 d / \alpha-1} \exp \left\{-\frac{1}{2}\left(\frac{u_{T}}{w\left(z_{1}\right)}\right)^{2}\right\} \\
& \quad \times\left(\int_{\left\{z_{2}: w\left(z_{2}\right)>w\left(z_{1}\right)\right\}}\left(1+\frac{w\left(z_{1}\right)}{w\left(z_{2}\right)}\right)^{2 d / \alpha-1}\left(1+\frac{w\left(z_{2}\right)}{w\left(z_{1}\right)}\right)^{2 d / \alpha}\left(\frac{u_{T}}{w\left(z_{2}\right)}\right)^{2 d / \alpha}\right. \\
& \leqslant \frac{(1+\varepsilon) H_{\alpha, \alpha} \cdot 2^{2 d / \alpha+3 / 2} T^{2 d}}{H_{\alpha}^{2} \sqrt{2 \pi}} \\
& \times \int_{\mathbf{R}^{d}} \Phi_{d, \alpha}\left(\frac{u_{T}}{w\left(z_{1}\right)}\right)\left(\int_{\left\{z_{2}: w\left(z_{2}\right)>w\left(z_{1}\right)\right\}}\left(1+\frac{w\left(z_{2}\right)}{w\left(z_{1}\right)}\right)^{2 d / \alpha} \Phi_{d, \alpha}\left(\frac{u_{T}}{w\left(z_{2}\right)}\right)\right. \\
& \left.\times \frac{u_{T}}{w\left(z_{2}\right)} \exp \left\{-\frac{w\left(z_{2}\right)}{2 w^{2}\left(z_{2}\right)}\left(\frac{w\left(z_{2}\right)}{w\left(z_{1}\right)}-1\right)\right\} d z_{2}\right) d z_{1},
\end{aligned}
$$

где мы воспользовались симметрией выражения по отношению к $z_{1}, z_{2}$ В (15).

Заметим, что, подобно шагу 1 в [4], можно показать, что главный вклад в интегралы дают множества $\left\{z_{i}: w\left(z_{i}\right)>c\right\}$ для любых $0<c<1$, 
$i=1,2$ при $T \rightarrow \infty$; точнее, интегралы по множествам $\left\{z_{i}: w\left(z_{i}\right) \leqslant c\right\}$ для любых $0<c<1, i=1,2$ имеют меньший порядок при $T \rightarrow \infty$. Рассмотрим более подробно внутренний интеграл в (15) в случае, когда $w\left(z_{1}\right)>c:$

$$
\begin{aligned}
& \int_{\left\{z_{2}: w\left(z_{2}\right)>w\left(z_{1}\right)\right\}}\left(1+\frac{w\left(z_{2}\right)}{w\left(z_{1}\right)}\right)^{2 d / \alpha} \Phi_{d, \alpha}\left(\frac{u_{T}}{w\left(z_{2}\right)}\right) \frac{u_{T}}{w\left(z_{2}\right)} \\
& \quad \times \exp \left\{-\frac{u_{T}^{2}}{2 w^{2}\left(z_{2}\right)}\left(\frac{w\left(z_{2}\right)}{w\left(z_{1}\right)}-1\right)\right\} d z_{2} \\
& \leqslant \int_{\left\{z_{2}: w\left(z_{2}\right)>\left(1+\varepsilon_{1}\right) w\left(z_{1}\right)\right\}}\left(1+\frac{1}{c}\right)^{2 d / \alpha} \Phi_{d, \alpha}\left(\frac{u_{T}}{w\left(z_{2}\right)}\right) \frac{u_{T}}{c} \exp \left\{-\frac{u_{T}^{2} \varepsilon_{1}}{2}\right\} d z_{2} \\
& \quad+\int_{\left\{z_{2}:\left(1+\varepsilon_{1}\right) w\left(z_{1}\right) \geqslant w\left(z_{2}\right)>w\left(z_{1}\right)\right\}}\left(2+\varepsilon_{1}\right)^{2 d / \alpha} \Phi_{d, \alpha}\left(\frac{u_{T}}{w\left(z_{2}\right)}\right) \frac{u_{T}}{c} \\
& \times \Lambda_{d, \alpha}\left(u_{T}\right)\left(1+\frac{1}{c}\right)^{2 d / \alpha} \frac{u_{T}}{c} \exp \left\{-\frac{u_{T}^{2}}{2}\left(\frac{w\left(z_{2}\right)}{w\left(z_{1}\right)}-1\right)\right\} d z_{2}
\end{aligned}
$$

для любого $0<c<1$, любого $\varepsilon_{1}>0$, некоторой константы $C>0$ и всех достаточно больших $T$. Объединяя (15) и (16), мы имеем

$$
\begin{aligned}
& \sum \sum_{k \neq m \in \mathbf{Z}^{d}} \mathbf{P}\left\{\sup _{s \in L_{k}(h)} w_{T}(s)|X(s)|>u_{T}, \sup _{s \in L_{m}(h)} w_{T}(s)|X(s)|>u_{T}\right\} \\
& \leqslant C_{1}(1+\varepsilon) T^{2 d} \Lambda_{d, \alpha}^{2}\left(u_{T}\right) u_{T} \exp \left\{-\frac{u_{T}^{2} \varepsilon_{1}}{2}\right\}\left(1+C_{2} \varepsilon_{1}\right) \leqslant e^{-2 t} o_{T}(1)
\end{aligned}
$$

при $T \rightarrow \infty$ ввиду этапа 1 .

6b. Покажем, что при $T \rightarrow \infty$

$$
\sum_{k \in \mathbf{Z}^{d}} \mathbf{P}\left\{\sup _{s \in L_{k}(h)} w_{T}(s)|X(s)|>u_{T}\right\} \geqslant e^{-t}\left(1+o_{T}(1)\right) .
$$

Данную одинарную сумму можно оценить следующим образом:

$$
\begin{aligned}
& \sum_{k \in \mathbf{Z}^{d}} \mathbf{P}\left\{\sup _{s \in L_{k}(h)} w_{T}(s)|X(s)|>u_{T}\right\} \\
& \geqslant \sum_{k \in \mathbf{Z}^{d}} \sum_{m \in M_{k}(h, \delta)} \mathbf{P}\left\{\sup _{s \in Q_{k, m}(h, \delta)} w_{T}(s)|X(s)|>u_{T}\right\} \\
&-\sum_{k \in \mathbf{Z}^{d}} \sum_{m \neq l \in M_{k}(h, \delta)} \mathbf{P}\left\{\sup _{s \in Q_{k, m}(h, \delta)} w_{T}(s)|X(s)|>u_{T},\right. \\
& \geqslant\left.\sum_{k \in \mathbf{Z}^{d}} \sum_{m \in M_{k}(h, \delta)} \mathbf{P u p} w_{s \in Q_{k, l}(h, \delta)} w_{T}(s)|X(s)|>u_{T}\right\} \\
&\left.\sup _{s \in Q_{k, m}(h, \delta)}|X(s)|>\frac{u_{T}}{w\left(\tilde{m}_{k}^{(h, \delta)} / T\right)}\right\}
\end{aligned}
$$




$$
\begin{array}{r}
-\sum_{k \in \mathbf{Z}^{d}} \sum_{m \neq l \in M_{k}(h, \delta)} \sum_{m \in Q_{k, m}(h, \delta)}|X(s)|>\frac{u_{T}}{w\left(m_{k}^{(h, \delta)} / T\right)}, \\
\left.\sup _{s \in Q_{k, l}(h, \delta)}|X(s)|>\frac{u_{T}}{w\left(l_{k}^{(h, \delta)} / T\right)}\right\},
\end{array}
$$

где

$$
\begin{aligned}
w\left(\frac{m_{k}^{(h, \delta)}}{T}\right) & =\max _{l \in \mathbf{Z}^{d} \cap Q_{k, m}(h, \delta)} w\left(\frac{l}{T}\right), \\
w\left(\frac{\widetilde{m}_{k}^{(h, \delta)}}{T}\right) & =\min _{l \in \mathbf{Z}^{d} \cap Q_{k, m}(h, \delta)} w\left(\frac{l}{T}\right),
\end{aligned}
$$

положительны, поскольку мы работаем на носителе $W$ функции $w$.

Как и на этапе 5, покажем напрямую, что для любого $\varepsilon \in(0,1)$

$$
\begin{aligned}
& \sum_{k \in \mathbf{Z}^{d}} \sum_{m \in M_{k}(h, \delta)} \mathbf{P}\left\{\sup _{s \in Q_{k, m}(h, \delta)}|X(s)|>\frac{u_{T}}{w\left(\widetilde{m}_{k}^{(h, \delta)} / T\right)}\right\} \\
& \geqslant(1-\varepsilon) T^{d} \int_{\mathbf{R}^{d}} \Phi_{d, \alpha}\left(\frac{u_{T}}{w(z)}\right) d z=(1-\varepsilon) e^{-t}\left(1+o_{T}(1)\right) .
\end{aligned}
$$

Рассмотрение двойной суммы в (17) очень подобно этапу $6 \mathrm{a}$ со следующим осложнением. Дисперсия поля $Y\left(s_{1}, s_{2}\right)=X\left(s_{1}\right)+X\left(s_{2}\right)$, $\left(s_{1}, s_{2}\right) \in Q_{k, m}(h, \delta) \times Q_{k, l}(h, \delta)$, не равна 2 для индексов $m, l$ таких, что $(\|m-l\|+1) \delta<1$, соответствующих кубам, которые отстоят друг от друга менее чем на 1. Используя условие (R2), мы имеем

$$
\sup _{\left(s_{1}, s_{2}\right) \in Q_{k, m}(h, \delta) \times Q_{k, l}(h, \delta)} \sigma_{Y}^{2}\left(s_{1}, s_{2}\right) \leqslant 4 .
$$

Следовательно, как и на этапе 6а, мы имеем

$$
\begin{gathered}
\sum_{k \in \mathbf{Z}^{d}} \sum_{m \neq l \in M_{k}(h, \delta)} \mathbf{P}\left\{\sup _{s \in Q_{k, m}(h, \delta)}|X(s)|>\frac{u_{T}}{w\left(m_{k}^{(h, \delta)} / T\right)}\right. \\
\left.\sup _{s \in Q_{k, l}(h, \delta)}|X(s)|>\frac{u_{T}}{w\left(l_{k}^{(h, \delta)} / T\right)}\right\} \\
\leqslant \sum_{k \in \mathbf{Z}^{d}} \sum_{m \neq l \in M_{k}(h, \delta)} 2 \mathbf{P}\left\{\sup _{\left(s_{1}, s_{2}\right) \in Q_{k, m}(h, \delta) \times Q_{k, l}(h, \delta)} Y^{*}\left(s_{1}, s_{2}\right)\right. \\
\left.>\frac{1}{2} \frac{u_{T}}{w\left(m_{k}^{(h, \delta)} / T\right)}+\frac{1}{2} \frac{u_{T}}{w\left(l_{k}^{(h, \delta)} / T\right)}\right\}
\end{gathered}
$$

где $Y^{*}\left(s_{1}, s_{2}\right)=Y\left(s_{1}, s_{2}\right) / \sigma_{Y}\left(s_{1}, s_{2}\right)$ для $\left(s_{1}, s_{2}\right) \in Q_{k, m}(h, \delta) \times Q_{k, l}(h, \delta)$. Заметим, что

$\mathbf{E}\left(Y^{*}\left(s_{1}, s_{2}\right) Y^{*}\left(s_{1}^{\prime}, s_{2}^{\prime}\right)\right)=\frac{r\left(s_{1}-s_{1}^{\prime}\right)+r\left(s_{1}-s_{2}^{\prime}\right)+r\left(s_{1}^{\prime}-s_{2}\right)+r\left(s_{2}-s_{2}^{\prime}\right)}{\left(\left(2+2 r\left(s_{1}-s_{1}^{\prime}\right)\right)\left(2+2 r\left(s_{1}^{\prime}-s_{2}^{\prime}\right)\right)\right)^{1 / 2}}$ 
для любых $\left(s_{1}, s_{2}\right),\left(s_{1}^{\prime}, s_{2}^{\prime}\right) \in Q_{k, m}(h, \delta) \times Q_{k, l}(h, \delta)$. В силу непрерывности $r$ всюду для любых $s_{1}, s_{2}, s_{1}^{\prime}, s_{2}^{\prime}$ таких, что $\left\|s_{1}-s_{1}^{\prime}\right\| \leqslant \delta$ и $\left\|s_{2}-s_{2}^{\prime}\right\| \leqslant \delta$, мы имеем

$r\left(s_{1}-s_{2}^{\prime}\right) \geqslant r\left(s_{1}-s_{2}\right)-\varepsilon_{1}\left\|s_{2}-s_{2}^{\prime}\right\|^{\alpha}, \quad r\left(s_{1}^{\prime}-s_{2}\right) \geqslant r\left(s_{1}-s_{2}\right)-\varepsilon_{1}\left\|s_{1}-s_{1}^{\prime}\right\|^{\alpha}$

и

$$
r\left(s_{1}^{\prime}-s_{2}^{\prime}\right) \leqslant r\left(s_{1}-s_{2}\right)+\varepsilon_{2}\left(\left\|s_{1}-s_{1}^{\prime}\right\|^{\alpha}+\left\|s_{2}-s_{2}^{\prime}\right\|^{\alpha}\right)
$$

для всех достаточно малых $\delta>0$ и некоторых малых $\varepsilon_{1}, \varepsilon_{2}>0$. Для достаточно малого $\delta>0$ мы имеем

$$
1-2\|s\|^{\alpha} \leqslant r(s) \leqslant 1-\frac{\|s\|^{\alpha}}{2} \text { для всех } s \text { таких, что }\|s\| \leqslant \delta \text {. }
$$

Тогда для всех $s_{1}, s_{2}, s_{1}^{\prime}, s_{2}^{\prime}$ таких, что $\left\|s_{1}-s_{1}^{\prime}\right\| \leqslant \delta$ и $\left\|s_{2}-s_{2}^{\prime}\right\| \leqslant \delta$, имеет место оценка

$$
\begin{aligned}
& \frac{r\left(s_{1}-s_{1}^{\prime}\right)+r\left(s_{1}-s_{2}^{\prime}\right)+r\left(s_{1}^{\prime}-s_{2}\right)+r\left(s_{2}-s_{2}^{\prime}\right)}{\left(\left(2+2 r\left(s_{1}-s_{2}\right)\right)\left(2+2 r\left(s_{1}^{\prime}-s_{2}^{\prime}\right)\right)\right)^{1 / 2}} \\
\geqslant & \frac{r\left(s_{1}-s_{1}^{\prime}\right)+r\left(s_{1}-s_{2}^{\prime}\right)+r\left(s_{1}^{\prime}-s_{2}\right)+r\left(s_{2}-s_{2}^{\prime}\right)}{\left(2+2 r\left(s_{1}-s_{2}\right)\right)\left(1+\left(\varepsilon_{2}\left(\left\|s_{1}-s_{1}^{\prime}\right\|^{\alpha}+\left\|s_{2}-s_{2}^{\prime}\right\|^{\alpha}\right)\right) /\left(4+4 r\left(s_{1}-s_{2}\right)\right)\right)} \\
\geqslant & \frac{1}{\left(2+2 r\left(s_{1}-s_{2}\right)\right)\left(1+\left(\varepsilon_{2}\left(\left\|s_{1}-s_{1}^{\prime}\right\|^{\alpha}+\left\|s_{2}-s_{2}^{\prime}\right\|^{\alpha}\right)\right) /\left(4+4 r\left(s_{1}-s_{2}\right)\right)\right)} \\
& \times\left(1-2\left\|s_{1}-s_{1}^{\prime}\right\|^{\alpha}+2 r\left(s_{1}-s_{2}\right)\right. \\
\geqslant & \quad-\frac{\left(\varepsilon_{1}\left(\left\|s_{1}-s_{1}^{\prime}\right\|^{\alpha}+\left\|s_{2}-s_{2}^{\prime}\right\|^{\alpha}\right)+1-2\left\|s_{1}-s_{1}^{\prime}\right\|^{\alpha}\right)}{\left(2+2 r\left(s_{1}-s_{2}\right)\right)\left(1+\varepsilon_{2}\left(\left\|s_{1}-s_{1}^{\prime}\right\|^{\alpha}+\left\|s_{2}-s_{2}^{\prime}\right\|^{\alpha}\right) /\left(4+4 r\left(s_{1}-s_{2}\right)\right)\right)} \\
\geqslant & 1-\frac{2+\varepsilon_{1}+\varepsilon_{2} / 2}{2 \varepsilon_{3}}\left(\left\|s_{1}-s_{1}^{\prime}\right\|^{\alpha}+\left\|s_{2}-s_{2}^{\prime}\right\|^{\alpha}\right) \\
& \times\left(1-C_{0}\left(\left\|s_{1}-s_{1}^{\prime}\right\|^{\alpha}+\left\|s_{2}-s_{2}^{\prime}\right\|^{\alpha}\right)+C_{0}^{2}\left(\left\|s_{1}-s_{1}^{\prime}\right\|^{2 \alpha}+\left\|s_{2}-s_{2}^{\prime}\right\|^{2 \alpha}\right)\right),
\end{aligned}
$$

где мы использовали неравенство $\sqrt{1+t} \leqslant 1+t / 2$ для всех $t>-1$ в первой строке; мы также воспользовались тем, что $\sup _{\|s\|>\varepsilon}|r(s)|<1$ для всех $\varepsilon>0$, а $s_{1} \neq s_{2}$ таковы, что $r\left(s_{1}-s_{2}\right)>-1+\varepsilon_{3}$ для некоторого $\varepsilon_{3}>0$, и $C_{0}-$ подходящая положительная константа.

Подобно этапу 6а, пусть $Z_{1}(s), Z_{2}(s), s \in \mathbf{R}^{d}$, - два независимых центрированных однородных гауссовских поля с ковариационными функциями $r_{Z}\left(s_{1}, s_{2}\right)=\exp \left\{-2 C_{0}\left\|s_{1}-s_{2}\right\|^{\alpha}\right\}$. Тогда однородное гауссовское поле

$$
Z^{*}\left(s_{1}, s_{2}\right)=\frac{Z_{1}\left(s_{1}\right)+Z_{2}\left(s_{2}\right)}{\sqrt{2}}, \quad\left(s_{1}, s_{2}\right) \in Q_{k, i}(h, \delta) \times Q_{m, j}(h, \delta),
$$


обладает ковариацией

$$
\begin{aligned}
& \frac{1}{2}\left(\exp \left\{-2 C_{0}\left\|s_{1}-s_{1}^{\prime}\right\|^{\alpha}\right\}+\exp \left\{-2 C_{0}\left\|s_{2}-s_{2}^{\prime}\right\|^{\alpha}\right\}\right) \\
& \quad \leqslant 1-C_{0}\left\|s_{1}-s_{1}^{\prime}\right\|^{\alpha}+C_{0}^{2}\left\|s_{1}-s_{1}^{\prime}\right\|^{2 \alpha}-C_{0}\left\|s_{2}-s_{2}^{\prime}\right\|^{\alpha}+C_{0}^{2}\left\|s_{2}-s_{2}^{\prime}\right\|^{2 \alpha} \\
& \quad \leqslant \mathbf{E}\left(Y^{*}\left(s_{1}, s_{2}\right) Y^{*}\left(s_{1}^{\prime}, s_{2}^{\prime}\right)\right)
\end{aligned}
$$

для всех $\left(s_{1}, s_{2}\right),\left(s_{1}^{\prime}, s_{2}^{\prime}\right) \in Q_{k, i}(h, \delta) \times Q_{m, j}(h, \delta)$, поскольку $e^{-u} \leqslant 1-u+$ $u^{2} / 2$ для любого $u \geqslant 0$.

Затем по неравенству Слепяна (см., к примеру, [11, теорема С.1]) и лемме 2 с $k=2, \tau=(\alpha, \alpha)$ мы имеем

$$
\begin{aligned}
& \mathbf{P}\left\{\sup _{s \in Q_{k, m}(h, \delta)}|X(s)|>\frac{u_{T}}{w\left(m_{k}^{(h, \delta)} / T\right)}, \sup _{s \in Q_{k, l}(h, \delta)}|X(s)|>\frac{u_{T}}{w\left(l_{k}^{(h, \delta)} / T\right)}\right\} \\
& \leqslant 2 \mathbf{P}\left\{\sup _{\left(s_{1}, s_{2}\right) \in Q_{k, m}(h, \delta) \times Q_{k, l}(h, \delta)} Z^{*}\left(s_{1}, s_{2}\right)\right. \\
& \left.\quad>\frac{1}{\sqrt{2}}\left(\frac{u_{T}}{w\left(m_{k}^{(h, \delta)} / T\right)}+\frac{u_{T}}{w\left(l_{k}^{(h, \delta)} / T\right)}\right)\right\} \\
& \leqslant(1+\varepsilon) \cdot 2^{2 d / \alpha+1} \operatorname{meas}\left(Q_{k, m}(h, \delta) \times Q_{k, l}(h, \delta)\right) \\
& \quad \times H_{\alpha, \alpha}^{*}\left(\frac{1}{\sqrt{2}}\left(\frac{u_{T}}{w\left(m_{k}^{(h, \delta)} / T\right)}+\frac{u_{T}}{w\left(l_{k}^{(h, \delta)} / T\right)}\right)\right)^{4 d / \alpha-1} \\
& \quad \times \varphi\left(\frac{1}{\sqrt{2}}\left(\frac{u_{T}}{w\left(m_{k}^{(h, \delta)} / T\right)}+\frac{\left.u_{T}^{(h, \delta)}\right)}{w\left(l_{k}^{(h, \delta)}\right)}\right)\right.
\end{aligned}
$$

и

$$
\begin{gathered}
\sum_{k \in \mathbf{Z}^{d}} \sum_{m \neq l \in M_{k}(h, \delta)} \mathbf{P}\left\{\sup _{s \in Q_{k, m}(h, \delta)}|X(s)|>\frac{u_{T}}{w\left(m_{k}^{(h, \delta)} / T\right)},\right. \\
\left.\sup _{s \in Q_{k, l}(h, \delta)}|X(s)|>\frac{u_{T}}{w\left(l_{k}^{(h, \delta)} / T\right)}\right\} \\
\leqslant \sum_{k \in \mathbf{Z}^{d}} \sum_{m \neq l \in M_{k}(h, \delta)}(1+\varepsilon) \cdot 2^{2 d / \alpha+1} \delta^{2 d} \sum_{m} \sum_{\alpha}^{*}\left(\frac{1}{\sqrt{2}}\left(\frac{u_{T}}{w\left(m_{k}^{(h, \delta)} / T\right)}+\frac{u_{T}}{w\left(l_{k}^{(h, \delta)} / T\right)}\right)\right)^{4 d / \alpha-1} \\
\times \varphi\left(\frac{1}{\sqrt{2}}\left(\frac{u_{T}}{w\left(m_{k}^{(h, \delta)} / T\right)}+\frac{u_{T}^{(h)}}{w\left(l_{k}^{(h, \delta)} / T\right)}\right)\right),
\end{gathered}
$$

где $H_{\alpha}^{*}$ и $H_{\alpha, \alpha}^{*}$ определены подобно $H_{\alpha}$ и $H_{\alpha, \alpha}$ с константой 4 , замененной на $2 C_{0}$.

Как и на этапе 6a, мы имеем

$$
\sum_{k \in \mathbf{Z}^{d}} \sum \sum_{m \neq l \in M_{k}(h, \delta)} \mathbf{P}\left\{\sup _{s \in Q_{k, m}(h, \delta)}|X(s)|>\frac{u_{T}}{w\left(m_{k}^{(h, \delta)} / T\right)},\right.
$$




$$
\begin{gathered}
\left.\sup _{s \in Q_{k, l}(h, \delta)}|X(s)|>\frac{u_{T}}{w\left(l_{k}^{(h, \delta)} / T\right)}\right\} \\
\leqslant C(1+\varepsilon) \sum_{k \in \mathbf{Z}^{d}} \int_{L_{k}(h)} \int_{L_{k}(h)}\left(\frac{u_{T}}{w\left(y_{1} / T\right)}+\frac{u_{T}}{w\left(y_{2} / T\right)}\right)^{4 d / \alpha-1} \\
\times \varphi\left(\frac{1}{\sqrt{2}}\left(\frac{u_{T}}{w\left(y_{1} / T\right)}+\frac{u_{T}}{w\left(y_{2} / T\right)}\right)\right) d y_{1} d y_{2} \\
\leqslant C(1+\varepsilon) e^{-2 t} o_{T}(1)
\end{gathered}
$$

при $T$, стремящемся к бесконечности. На самом деле последнее неравенство очень грубое, но оно достаточно для наших целей. Можно доказать более быструю сходимость к 0.

7. Покажем, что для любого $\varepsilon>0$

$$
\ln \left(1-P_{1}\right) \geqslant-\frac{1+\varepsilon}{1-\varepsilon} e^{-t}\left(1+o_{T}(1)\right) \quad \text { при } T \rightarrow \infty .
$$

В силу условия (R2) у нас есть независимость, так что

$$
\begin{aligned}
1-P_{1} & =\mathbf{P}\left\{\max _{k \in \mathbf{Z}^{d}} \sup _{s \in L_{k}(h)} w_{T}(s)|X(s)| \leqslant u_{T}\right\} \\
& =\prod_{k \in \mathbf{Z}^{d}} \mathbf{P}\left\{\sup _{s \in L_{k}(h)} w_{T}(s) X(s) \leqslant u_{T}\right\} .
\end{aligned}
$$

Тогда

$$
\begin{aligned}
\ln \left(1-P_{1}\right) & =\sum_{k \in \mathbf{Z}^{d}} \ln \left(1-\mathbf{P}\left\{\sup _{s \in L_{k}(h)} w_{T}(s)|X(s)|>u_{T}\right\}\right) \\
& \geqslant-\sum_{k \in \mathbf{Z}^{d}} \frac{\mathbf{P}\left\{\sup _{s \in L_{k}(h)} w_{T}(s)|X(s)|>u_{T}\right\}}{1-\mathbf{P}\left\{\sup _{s \in L_{k}(h)} w_{T}(s)|X(s)|>u_{T}\right\}} \\
& \geqslant-\frac{1}{1-\varepsilon} \sum_{k \in \mathbf{Z}^{d}} \mathbf{P}\left\{\sup _{s \in L_{k}(h)} w_{T}(s)|X(s)|>u_{T}\right\},
\end{aligned}
$$

где мы применили простое неравенство $\ln (1-a) \leqslant a /(1-a)$ для $a \rightarrow 0$ с $a=\mathbf{P}\left\{\sup _{s \in L_{k}(h)} w_{T}(s)|X(s)|>u_{T}\right\} \rightarrow 0$ при $T \rightarrow \infty$. Таким образом, для любого $\varepsilon>0$ и для всех достаточно больших $T$ мы имеем (18). При помощи рассуждений этапа 5 мы получаем

$$
\sum_{k \in \mathbf{Z}^{d}} \mathbf{P}\left\{\sup _{s \in L_{k}(h)} w_{T}(s)|X(s)|>u_{T}\right\} \leqslant(1+\varepsilon) e^{-t}\left(1+o_{T}(1)\right) .
$$

Значит,

$$
\ln \left(1-P_{1}\right) \geqslant-\frac{1+\varepsilon}{1-\varepsilon} e^{-t}\left(1+o_{T}(1)\right)
$$

для любого $\varepsilon>0$ и всех достаточно больших $T$.

В результате этапов 4-7 мы имеем при $T \rightarrow \infty$

$$
\ln \left(1-P_{0}\right)=-e^{-t}\left(1+o_{T}(1)\right),
$$

что и завершает доказательство теоремы 1. 


\section{5. Другие доказательства.}

5.1. Доказательство предложения 1. Для всех $u>u_{0}$ имеем

$$
\begin{aligned}
\Lambda_{d, \alpha}(u) & =\int_{\mathbf{R}^{d}}\left(\frac{u}{u_{0}}\right)^{2 d / \alpha-1} \exp \left\{-\frac{u^{2}-u_{0}^{2}}{2 w^{2}(y)}\right\} \Psi_{d, \alpha}\left(\frac{u_{0}}{w(y)}\right) d y \\
& \leqslant\left(\frac{u}{u_{0}}\right)^{2 d / \alpha-1} \exp \left\{-\frac{u^{2}-u_{0}^{2}}{2 w_{\max }^{2}}\right\} \Lambda_{d, \alpha}\left(u_{0}\right) .
\end{aligned}
$$

Из определения $A_{T}$ следует, что $\ln \left(T^{d} \Lambda_{d, \alpha}\left(A_{T}\right)\right) \rightarrow 0$ при $T \rightarrow \infty$. Тогда

$$
\begin{aligned}
\ln \left(T^{d} \Lambda_{d, \alpha}\left(A_{T}\right)\right) \leqslant & d \ln T+\left(\frac{2 d}{\alpha-1}\right)\left(\ln A_{T}-\ln u_{0}\right) \\
& -\frac{A_{T}^{2}-u_{0}^{2}}{2 \sup _{x \in \mathbf{R}^{d}} w(x)^{2}}+\ln \Lambda_{d, \alpha}\left(u_{0}\right),
\end{aligned}
$$

и поэтому $A_{T}$ не может расти быстрее, чем $\sup _{x \in \mathbf{R}^{d}} w(x) \sqrt{2 d \ln T}(1+o(1))$ в асимптотике.

С другой стороны, для любого $\varepsilon>0$

$$
\begin{aligned}
\Lambda_{d, \alpha}(u)= & \int_{w(y) \geqslant \sup _{x \in \mathbf{R}^{d}} w(x)-\varepsilon} \Psi_{d, \alpha}\left(\frac{u}{w(y)}\right) d y \\
& +\int_{w(y)<\sup _{x \in \mathbf{R}^{d}} w(x)-\varepsilon} \Psi_{d, \alpha}\left(\frac{u}{w(y)}\right) d y \\
\geqslant & \frac{2}{\sqrt{2 \pi}} H_{\alpha} C_{r}^{1 / \alpha}\left(\frac{u}{\sup _{x \in \mathbf{R}^{d}} w(x)}\right)^{2 d / \alpha-1} \\
& \times \exp \left\{-\frac{u^{2}}{2\left(\sup _{x \in \mathbf{R}^{d}} w(x)-\varepsilon\right)^{2}}\right\} \int_{\left\{y: w(y) \geqslant \sup _{x \in \mathbf{R}^{d}} w(x)-\varepsilon\right\}} d y,
\end{aligned}
$$

откуда следует, что $A_{T}$ не может расти медленнее, чем

$$
\left(\sup _{x \in \mathbf{R}^{d}} w(x)-\varepsilon\right)\left\{2 d\left(\ln T+o(\ln T)+\ln \int_{\left.w(y) \geqslant \sup _{x \in \mathbf{R}^{d}} w(x)-\varepsilon\right)} d y\right\}^{1 / 2}\right.
$$

при $T \rightarrow \infty$. Используя условие (w1), выберем $\varepsilon=\varepsilon_{T}$ стремящимся к 0 при $T \rightarrow \infty$ так медленно, чтобы выполнялось соотношение

$$
\ln \int_{\left\{y: w(y) \geqslant \sup _{x \in \mathbf{R}^{d}} w(x)-\varepsilon_{T}\right\}} d y=o(\ln T),
$$

это и завершает доказательство предложения.

5.2. Доказательство теорем 2 и 3. Мы придерживаемся схемы доказательства теоремы 6 в [4]. Обозначим $\lambda_{t}=\left(t h_{t}^{d}\left|\ln h_{t}\right|\right)^{1 / 2}$.

Как и в доказательстве теоремы 2.1 в [5], разобьем множество $B_{f}$ на 4 непересекающихся подмножества:

$$
B_{f}=D_{a} \cup \mathscr{A}_{n} \cup \mathscr{B}_{n} \cup \mathscr{C}_{n, a}
$$


для фиксированного $a>0$, где

$$
\begin{aligned}
\mathscr{A}_{n} & :=\left\{x \in B_{f}: \Psi(x)>\lambda_{n \ln n}\right\}, \\
\mathscr{B}_{n} & :=\left\{x \in B_{f}: f(x) \Psi(x) \leqslant(\ln n)^{-(1-\beta)}\left(\frac{\left|\ln h_{n}\right|}{n h_{n}^{d}}\right)^{1 / 2}, \Psi(x) \leqslant \lambda_{n \ln n}\right\}, \\
\mathscr{C}_{n, a} & :=\left\{x \in B_{f} \cap D_{a}^{c}: f(x) \Psi(x)>(\ln n)^{-(1-\beta)}\left(\frac{\left|\ln h_{n}\right|}{n h_{n}^{d}}\right)^{1 / 2}\right\} .
\end{aligned}
$$

Тогда, если выполнено условие (5) и если либо $B_{f}=\mathbf{R}^{d}$, либо $K(0)=$ $\|K\|_{\infty}$, то мы можем соединить результаты $(2.8),(2.10),(2.20)$ в [5] с леммой 5 в [4], утверждающей, что

$$
\limsup _{n \rightarrow \infty}\left(A_{1 / h_{n}}+\frac{t}{A_{1 / h_{n}}}\right)\left|\ln h_{n}\right|^{-1 / 2}<\infty
$$

для всех $t \in \mathbf{R}$, и получить

$$
\begin{aligned}
& \lim _{n \rightarrow \infty} \mathbf{P}\left\{A_{1 / h_{n}}\left(\sqrt{n h_{n}^{d}}\|K\|_{2}^{-1} \sup _{x \in \mathscr{A}_{n}}\left|\Psi(x)\left(\widehat{f}_{n}(x)-\mathbf{E} \widehat{f}_{n}(x)\right)\right|-A_{1 / h_{n}}\right) \geqslant t\right\}=0, \\
& \lim _{n \rightarrow \infty} \mathbf{P}\left\{A_{1 / h_{n}}\left(\sqrt{n h_{n}^{d}}\|K\|_{2}^{-1} \sup _{x \in \mathscr{B}_{n}}\left|\Psi(x)\left(\widehat{f}_{n}(x)-\mathbf{E} \widehat{f}_{n}(x)\right)\right|-A_{1 / h_{n}}\right) \geqslant t\right\}=0
\end{aligned}
$$

и

$$
\begin{gathered}
\lim _{a \rightarrow \infty} \limsup _{n \rightarrow \infty} \mathbf{P}\left\{A _ { 1 / h _ { n } } \left(\sqrt{n h_{n}^{d}}\|K\|_{2}^{-1} \sup _{x \in \mathscr{C}_{n, a}}\left|\Psi(x)\left(\widehat{f}_{n}(x)-\mathbf{E} \widehat{f}_{n}(x)\right)\right|\right.\right. \\
\left.\left.-A_{1 / h_{n}}\right) \geqslant t\right\}=0
\end{gathered}
$$

для всех $t \in \mathbf{R}$. Теорема 2 и следствие 4 в [4], утверждающие, что можно заменить супремум по всему пространству на супремум по $D_{a}$ без изменений в центрировании и нормировке $A_{1 / h_{n}}$, влекут за собой соотношение

$$
\begin{gathered}
\lim _{n \rightarrow \infty} \mathbf{P}\left\{A_{1 / h_{n}}\left(\sqrt{n h_{n}^{d}}\|K\|_{2}^{-1} \sup _{x \in D_{a}}\left|\Psi(x)\left(\widehat{f}_{n}(x)-\mathbf{E} \widehat{f}_{n}(x)\right)\right|-A_{1 / h_{n}}\right) \leqslant t\right\} \\
=\exp \left\{-e^{-t}\right\}
\end{gathered}
$$

для всех $t \in \mathbf{R}$ и всех достаточно больших $a>0$. Это доказывает достаточность условия $\lim _{t \rightarrow \infty} t \mathbf{P}\left\{\Psi\left(X_{1}\right)>\lambda_{t}\right\}=0$. Его необходимость может быть показана способом, аналогичным одномерному случаю в [4] (см. с. 189), что и завершает доказательство теоремы 2.

Доказательство теоремы 3 легко получается из доказательств теорем 8 и 9 в [4]. 


\section{6. Пример с выбираемыми по данным центрированием и} нормировкой. Использование теоремы 2 в приложениях, таких как доверительные полосы для плотностей, наталкивается на трудности. Вопервых, центрирование и нормировка $A_{T}$ зависит от $\sup _{x \in \mathbf{R}^{d}} \Psi(x) \sqrt{f(x)}$. Во-вторых, функция $\Psi$ тоже может зависеть от плотности $f$. В-третьих, нужно учитывать смещение $\widetilde{f}_{n}(x)-f(x):=\mathbf{E} \widehat{f}_{n}(x)-f(x), x \in \mathbf{R}^{d}$. В данном разделе мы отвечаем на эти трудности.

Во-первых, заметим, что теорема 1 выполняется с последовательностью $\widetilde{A}_{T}$, удовлетворяющей

$$
\lim _{T \rightarrow \infty} T^{d} \Lambda_{d, \alpha}\left(\widetilde{A}_{T}\right)=1
$$

что легко видеть из доказательства.

Во-вторых, отметим, что если $\left|\widetilde{A}_{T} / A_{T}-1\right|=o\left(A_{T}^{-2}\right)$, то

$$
\lim _{T \rightarrow \infty} T^{d} \Lambda_{d, \alpha}\left(\widetilde{A}_{T}\right)=1 .
$$

Сие наблюдение было сделано в одномерном случае в [4]. В многомерном случае оно весьма похоже. Действительно, пусть $\varepsilon_{T}=\left|\widetilde{A}_{T} / A_{T}-1\right|$. Рассмотрим для любого $c \in\left(0, \sup _{x \in \mathbf{R}^{d}} w(x)\right]$

$$
\begin{aligned}
\left(1+\varepsilon_{T}\right)^{2 d / \alpha-1} \geqslant & T^{d} \Lambda_{d, \alpha}\left(\widetilde{A}_{T}\right) \\
= & \left(1+\varepsilon_{T}\right)^{2 d / \alpha-1} \frac{\int_{W}\left(\frac{A_{T}}{w(y)}\right)^{2 d / \alpha-1} \exp \left\{-\frac{A_{T}^{2}}{2 w^{2}(y)}\left(1+\varepsilon_{T}\right)^{2}\right\} d y}{\int_{W}\left(\frac{A_{T}}{w(y)}\right)^{2 d / \alpha-1} \exp \left\{-\frac{A_{T}^{2}}{2 w^{2}(y)}\right\} d y} \\
\geqslant & \left(1+\varepsilon_{T}\right)^{2 d / \alpha-1} \exp \left\{-\frac{A_{T}^{2} \varepsilon_{T}\left(2+\varepsilon_{T}\right)}{2 c^{2}}\right\} \\
& \times \frac{\int_{\{y: w(y)>c\}}\left(\frac{A_{T}}{w(y)}\right)^{2 d / \alpha-1} \exp \left\{-\frac{A_{T}^{2}}{2 w^{2}(y)}\right\} d y}{\int_{W}\left(\frac{A_{T}}{w(y)}\right)^{2 d / \alpha-1} \exp \left\{-\frac{A_{T}^{2}}{2 w^{2}(y)}\right\} d y} .
\end{aligned}
$$

Тогда, если $\varepsilon_{T}=o\left(A_{T}^{-2}\right)$, то, устремляя $c$ к 0 , мы получим желаемую сходимость к 1.

Соединяя эти два факта с предложением 1 , можно заменить $A_{T}$ на

$$
B_{T}:=\sup _{x \in \mathbf{R}^{d}} w(x) \sqrt{2 d \ln T}+\left(\frac{d}{\alpha}-\frac{1}{2}\right) \sup _{x \in \mathbf{R}^{d}} w(x) \frac{\ln \ln T}{\sqrt{2 d \ln T}}
$$

в теореме 1 и, следовательно, заменить $A_{1 / h_{n}}$ на $B_{1 / h_{n}}$ в теореме 2 . Использование $B_{T}$ - более практично.

Введем дополнительные определения и дополнительные условия. Обозначим

$$
\Delta_{n}=\sup _{x \in \mathbf{R}^{d}} \max \left[\left|\tilde{f}_{n}(x)-f(x)\right|,\left|\nabla \tilde{f}_{n}(x)-\nabla f(x)\right|\right], \quad n \geqslant 1
$$


где $\nabla g$ обозначает градиент функции $g$. Пусть $\omega(u, x)$ - вещественнозначная функция, определенная для $u \in \mathbf{R}_{+}, x \in \mathbf{R}^{d}$, такая, что функция $w(x)=\omega(f(x), x), x \in \mathbf{R}^{d}$, удовлетворяет условиям (w1)-(w3), а функция $\Psi(x)=\omega(f(x), x) f^{-1 / 2}(x), x \in \mathbf{R}^{d}$, удовлетворяет условиям (W.a)-(W.c) и $(\mathrm{WD} . \mathrm{a})_{\beta}$. Обозначим

$$
\mathscr{M}=\left\{\tau \in \mathbf{R}^{d}: \omega(f(\tau), \tau)=\sup _{x \in \mathbf{R}^{d}} \omega(f(x), x)\right\}
$$

Заметим, что в силу (w1) множество $\mathscr{M}$ компактно и содержится в $D_{a}$ для всех достаточно больших $a>0$.

Далее, для всех достаточно малых $\delta>0$ замкнутая выпуклая оболочка $\delta$-окрестности множества $\mathscr{M}$ является компактом. Мы обозначим его $\mathscr{K}$. Определим $W_{a}=\{|x| \leqslant a, \omega(f(x), x) \geqslant 1 / a\}$ для $a>0$.

Введем следующие условия.

(А0) $\mathscr{K} \subset W$.

(A1) Функция $\omega$ дважды непрерывно дифференцируема на $\mathbf{R}^{d+1}$ и существует $\varepsilon^{*}>0$ такое, что

$$
\begin{aligned}
& \sup _{x \in \mathscr{K} f(x)-\varepsilon^{*} \leqslant u \leqslant f(x)+\varepsilon^{*}} \sup _{\sup _{x \in \mathscr{K}} f(x)-\varepsilon^{*} \leqslant u \leqslant f(x)+\varepsilon^{*}} \sup _{\sup \left|\frac{\partial \omega(u, x)}{\partial u}\right|<\infty,} \mid<\infty, \\
& \sup _{x \in \mathscr{K} f(x)-\varepsilon^{*} \leqslant u \leqslant f(x)+\varepsilon^{*}}\left|\frac{\partial^{2} \omega(u, x)}{\partial u \partial x}\right|<\infty .
\end{aligned}
$$

(A2) Для всех достаточно больших $a>0, n$ и всех $x \in D_{a}$

$$
\left|\omega\left(\widehat{f}_{n}(x), x\right)-\omega(f(x), x)\right| \leqslant C_{\omega}(a)\left|\widehat{f}_{n}(x)-f(x)\right|,
$$

где $C_{\omega}: \mathbf{R}_{+} \rightarrow \mathbf{R}_{+}$- ограниченная функция.

(А3) Для всех достаточно больших $a>0$ имеем $\inf _{x \in D_{a}} \omega(f(x), x) \geqslant$ $C_{0} a^{-\delta_{0}}$ с некоторыми константами $C_{0}>0$ и $\delta_{0}>0$.

Заметим, что условия (A3) и (WD.a) $\beta$ гарантируют, что $D_{a} \subset$ $W_{a^{*}} \subset D_{k a^{2 \delta_{0} /(1-2 \beta)}}$ для всех достаточно больших $a>0$, где $a^{*}=$ $1 / \inf _{x \in D_{a}} \omega(f(x), x)$ и $k=\left(\left\|\Psi f^{\beta}\right\|_{\infty} / C_{0}\right)^{2 /(1-2 \beta)}$. Условие (А0) выполнено, если $W=\mathbf{R}^{d}$.

Определим $\widehat{D}_{a}=\left\{|x| \leqslant a, \widehat{f}_{n}(x) \geqslant 1 / a\right\}$ и $\widehat{W}_{a}=\left\{|x| \leqslant a, \omega\left(\widehat{f}_{n}(x), x\right) \geqslant\right.$ $1 / a\}$ для $a>0$. Пусть

$$
\begin{aligned}
& B_{n}=\sup _{x \in \mathbf{R}^{d}} \omega(f(x), x)\left[\sqrt{2 d\left|\ln h_{n}\right|}+\left(\frac{d}{\alpha}-\frac{1}{2}\right) \frac{\ln \left|\ln h_{n}\right|}{\sqrt{2 d\left|\ln h_{n}\right|}}\right], \\
& \widehat{B}_{n}=\sup _{x \in \mathbf{R}^{d}} \omega\left(\widehat{f}_{n}(x), x\right)\left[\sqrt{2 d\left|\ln h_{n}\right|}+\left(\frac{d}{\alpha}-\frac{1}{2}\right) \frac{\ln \left|\ln h_{n}\right|}{\sqrt{2 d\left|\ln h_{n}\right|}}\right] .
\end{aligned}
$$


Теорема 5. Пусть выполнень условия теоремь 2, включая условие

$$
\lim _{t \rightarrow \infty} t \mathbf{P}\left\{\Psi\left(X_{1}\right)>\left(t h_{t}^{d}\left|\ln h_{t}\right|\right)^{1 / 2}\right\}=0 .
$$

Пусть также выполнень условия (A0)-(А3) $и h_{n}^{d+4} \rightarrow 0$ рри $n \rightarrow \infty$. Более того, предположим, что ядро $K$ дважды непрерьвно дифферениируемо на своем носителе $u f$ дифференцируема на $B_{f}$. Наконеи, пусть последовательности $a_{n} \rightarrow \infty u \Delta_{n} \stackrel{P}{\longrightarrow} 0, n \rightarrow \infty$, таковы, что

$$
\left|\ln h_{n}\right| \Delta_{n} \rightarrow 0, \quad\left[a_{n}^{1+\delta_{0}} \vee C_{\omega}\left(k a_{n}^{2 \delta_{0} /(1-2 \beta)}\right)\right]\left[\varepsilon_{n}\left(a_{n}\right)+\Delta_{n}\right] \stackrel{P}{\longrightarrow} 0
$$

при $n \rightarrow \infty$, где $\varepsilon_{n}(a)=a_{n}^{0.5+\delta_{0}} \sqrt{\left|\ln h_{n}\right| /\left(n h_{n}^{d}\right)}$. Тогда для всех $t \in \mathbf{R}$ миц имеем

$$
\begin{gathered}
\mathbf{P}\left\{\widehat{B}_{n}\left(\frac{\sqrt{n h_{n}^{d}}}{\|K\|_{2}} \sup _{x \in \widehat{D}_{a_{n}}}\left|\omega\left(\widehat{f}_{n}(x), x\right) \widehat{f}_{n}^{-1 / 2}(x)\left(\widehat{f}_{n}(x)-\widetilde{f}_{n}(x)\right)\right|-\widehat{B}_{n}\right) \leqslant t\right\} \\
\rightarrow \exp \left\{-e^{-t}\right\}
\end{gathered}
$$

при $n \rightarrow \infty$. Если дополнительно мьи имеем при $n \rightarrow \infty$

$$
\sqrt{n h_{n}^{d}\left|\ln h_{n}\right|} a_{n}^{\beta} \Delta_{n} \stackrel{P}{\longrightarrow} 0
$$

то в предьдущем утверждении о сходимости по распределению $\tilde{f}_{n}$ можно заменить на $f$.

Теорема 5 позволяет строить доверительные полосы для плотностей $f$, используя только выборку $\left(X_{1}, \ldots, X_{n}\right)$, информацию о структуре весовой функции $w$, выбор ширины окна $h_{n}$, уровень урезания $a_{n}$, ядро $K$ и ограничения на смещение. Конечно, данный результат - это всего лишь скромный шаг вперед, поскольку, например, можно пытаться выбирать $h_{n}, a_{n}$ и $K$ по данным с целью минимизации локальной ширины доверительной полосы.

6.1. Доказательство теоремы 5. Мы можем применить теорему 2 и получить

$$
\mathbf{P}\left\{A_{1 / h_{n}}\left(\frac{\sqrt{n h_{n}^{d}}}{\|K\|_{2}}\left\|\Psi(\cdot)\left(\widehat{f}_{n}(\cdot)-\widetilde{f}_{n}(\cdot)\right)\right\|_{\infty}-A_{1 / h_{n}}\right) \leqslant t\right\} \rightarrow \exp \left\{-e^{-t}\right\}
$$

при $n \rightarrow \infty$. Более того, в доказательстве теоремы 2 мы показали, что важен супремум только по $D_{a}$ для достаточно больших $a>0$. Тогда план доказательства состоит в следующем. Нам требуется доказать, что супремум можно брать по $\widehat{D}_{a_{n}}$ вместо $D_{a}$, что $A_{1 / h_{n}}$ можно заменить на $\widehat{B}_{n}$, что $\Psi(\cdot)$ можно поменять на $\omega\left(\widehat{f}_{n}(\cdot), \cdot\right) \widehat{f}_{n}(\cdot)^{-1 / 2}$ и что смещение можно проигнорировать. Для простоты изложения мы поделили остальное доказательство на шаги. 
1. Заметим, что для всех $x \in \mathbf{R}^{d}$ выполнены неравенства $f(x)-\Delta_{n} \leqslant$ $\tilde{f}_{n}(x) \leqslant f(x)+\Delta_{n}$ и

$$
\frac{\partial}{\partial x_{i}} f(x)-\Delta_{n} \leqslant \frac{\partial}{\partial x_{i}} \tilde{f}_{n}(x) \leqslant \frac{\partial}{\partial x_{i}} f(x)+\Delta_{n}, \quad i=1, \ldots, d .
$$

Заметим также, что для достаточно больших $a>0$ и для любых $x \in D_{a}$ мы имеем

$$
\begin{aligned}
\omega(f(x), x) f^{-1 / 2}(x) \tilde{f}_{n}(x) & \geqslant \inf _{s \in D_{a}} \omega(f(s), s)\left(f(x)-\Delta_{n}\right) f^{-1 / 2}(x) \\
& \geqslant \inf _{s \in D_{a}} \omega(f(s), s)\left(a^{-1 / 2}-\Delta_{n} \sqrt{a}\right) .
\end{aligned}
$$

Поскольку $\Delta_{n} a_{n} \rightarrow 0$ при $n \rightarrow \infty$, то $\omega(f(x), x) f^{-1 / 2}(x) \tilde{f}_{n}(x) \geqslant$ $C_{1} \inf _{s \in D_{a_{n}}} \omega(f(s), s) a_{n}^{-1 / 2}$ для всех $x \in D_{a_{n}}$ при $n \rightarrow \infty$.

Тогда

$$
\begin{aligned}
\sup _{x \in D_{a_{n}}}\left|\frac{\widehat{f}_{n}(x)-\tilde{f}_{n}(x)}{\widetilde{f}_{n}(x)}\right|=\sup _{x \in D_{a_{n}}}\left|\frac{\omega(f(x), x) f^{-1 / 2}(x)\left(\widehat{f}_{n}(x)-\widetilde{f}_{n}(x)\right)}{\omega(f(x), x) f^{-1 / 2}(x) \widetilde{f}_{n}(x)}\right| \\
\leqslant \frac{\sup _{x \in D_{a_{n}}}\left|\omega(f(x), x) f^{-1 / 2}(x)\left(\widehat{f}_{n}(x)-\widetilde{f}_{n}(x)\right)\right|}{C_{1} \inf _{s \in D_{a_{n}}} \omega(f(s), s)} a_{n}^{1 / 2} \\
=\frac{\sqrt{\left|\ln h_{n}^{d}\right|} a_{n}^{1 / 2+\delta_{0}} O_{P}(1)}{C_{1} C_{0} \sqrt{n h_{n}^{d}}} \leqslant C_{2} \varepsilon_{n}\left(a_{n}\right)
\end{aligned}
$$

при $n \rightarrow \infty$ по теореме 2.1 в [5] для некоторой положительной константы $C_{2}$. Определим событие

$$
E_{n}^{(1)}=\left\{\forall x \in D_{a_{n}}-C_{2} \varepsilon_{n}\left(a_{n}\right) \leqslant \frac{\widehat{f}_{n}(x)-\widetilde{f}_{n}(x)}{\widetilde{f}_{n}(x)} \leqslant C_{2} \varepsilon_{n}\left(a_{n}\right)\right\} .
$$

Тогда $P\left(E_{n}^{(1)}\right) \rightarrow 1$ при $n \rightarrow \infty$.

На событии $E_{n}^{(1)}$ мы имеем

$$
\begin{aligned}
\widehat{f}_{n}(x) \geqslant \frac{1}{a_{n}} & \Rightarrow \widetilde{f}_{n}(x) \geqslant \frac{\widehat{f}_{n}(x)}{1+C_{2} \varepsilon_{n}\left(a_{n}\right)} \geqslant \frac{1}{a_{n}\left(1+C_{2} \varepsilon_{n}\left(a_{n}\right)\right)} \\
& \Rightarrow f(x) \geqslant \widetilde{f}_{n}(x)-\Delta_{n} \geqslant \frac{1}{a_{n}\left(1+C_{2} \varepsilon_{n}\left(a_{n}\right)\right)}-\Delta_{n}
\end{aligned}
$$

и

$$
\begin{aligned}
\left(\frac{a_{n}\left(1-C_{2} \varepsilon_{n}\left(a_{n}\right)\right.}{1+a_{n} \Delta_{n}\left(1-C_{2} \varepsilon_{n}\left(a_{n}\right)\right)}\right)^{-1} & \leqslant f(x) \leqslant \tilde{f}_{n}(x)+\Delta_{n} \\
& \leqslant \frac{\widehat{f}_{n}(x)}{1-C_{2} \varepsilon_{n}\left(a_{n}\right)}+\Delta_{n} \Rightarrow \widehat{f}_{n}(x) \geqslant \frac{1}{a_{n}}
\end{aligned}
$$


Таким образом, на событии $E_{n}^{(1)}$ мы имеем

$$
\begin{aligned}
D_{a_{n}\left(1-C_{2} \varepsilon_{n}\left(a_{n}\right)\right) /\left(1+a_{n} \Delta_{n}\left(1-C_{2} \varepsilon_{n}\left(a_{n}\right)\right)\right)} & \subset \widehat{D}_{a_{n}} \\
& \subset D_{a_{n}\left(1+C_{2} \varepsilon_{n}\left(a_{n}\right)\right) /\left(1-a_{n} \Delta_{n}\left(1+C_{2} \varepsilon_{n}\left(a_{n}\right)\right)\right)},
\end{aligned}
$$

откуда следует, что $D_{a_{n}}$ можно заменить на $\widehat{D}_{a_{n}}$ в $(19)$.

2. Анализируя доказательство теоремы 2 , нам надо

$$
\limsup _{n \rightarrow \infty} \frac{\widehat{B}_{n}+t / \widehat{B}_{n}}{\sqrt{\left|\ln h_{n}^{d}\right|}}<\infty \quad \forall t \in \mathbf{R}
$$

для того, чтобы можно было заменить $\sqrt{\left|\ln h_{n}^{d}\right|}$ на $\widehat{B}_{n}+t / \widehat{B}_{n}$ для множеств $\mathscr{B}_{n}, \mathscr{A}_{n}, \mathscr{C}_{n, a}$. Более того, $B_{n}$ и $\widehat{B}_{n}$ удовлетворяют условиям для $\alpha_{n}$ в теореме 1 . Нам только надо проверить, что

$$
\left|\frac{\widehat{B}_{n}}{A_{1 / h_{n}}}-1\right|=o_{P}\left(A_{1 / h_{n}}^{-2}\right) \quad \text { при } n \rightarrow \infty ;
$$

для этого в силу предложения 1 достаточно показать, что

$$
\left|\frac{\widehat{B}_{n}}{B_{n}}-1\right|=o_{P}\left(B_{n}^{-2}\right) \quad \text { при } n \rightarrow \infty
$$

или, что эквивалентно,

$$
\left|\ln h_{n}\right|\left(\sup _{x \in \mathbf{R}^{d}} \omega\left(\widehat{f}_{n}(x), x\right)-\sup _{x \in \mathbf{R}^{d}} \omega(f(x), x)\right)=o_{P}(1) \quad \text { при } n \rightarrow \infty .
$$

$\mathrm{C}$ этой целью заметим, что для любого $\varepsilon>0$ существует $\delta>0$ такое, что при всех $t \notin \mathscr{M}_{\delta}$ мы имеем

$$
\omega(f(x), x) \leqslant \sup _{s \in W_{a}} \omega(f(s), s)-\varepsilon
$$

для любого достаточно большого $a>0$. Пусть

$$
\delta(\varepsilon)=\inf \left\{\delta>0: \forall t \notin \mathscr{M}_{\delta} \omega(f(x), x) \leqslant \sup _{s \in W_{a}} \omega(f(s), s)-\varepsilon\right\} .
$$

Тогда, рассуждая от противного, легко видеть, что $\delta(\varepsilon) \rightarrow 0$ при $\varepsilon \rightarrow 0$.

Определим событие

$$
E_{n, a}^{(2)}(\varepsilon)=\left\{\sup _{x \in W_{a}}\left|\omega\left(\widehat{f}_{n}(x), x\right)-\omega(f(x), x)\right| \leqslant \frac{\varepsilon}{3}\right\} .
$$

Принимая во внимание условие (А2) и замечание после условия (A3), мы имеем

$$
\begin{aligned}
\sup _{x \in W_{a_{n}}}\left|\omega\left(\widehat{f}_{n}(x), x\right)-\omega(f(x), x)\right| \leqslant C_{\omega}\left(k a_{n}^{2 \delta_{0} /(1-2 \beta)}\right) \sup _{x \in W_{a_{n}}}\left|\widehat{f}_{n}(x)-f(x)\right| \\
\leqslant C_{\omega}\left(k a_{n}^{2 \delta_{0} /(1-2 \beta)}\right)\left[\sup _{x \in W_{a_{n}}}\left|\widetilde{f}_{n}(x)\right| C_{2} \varepsilon_{n}\left(a_{n}\right)+\Delta_{n}\right] \\
\leqslant C_{\omega}\left(k a_{n}^{2 \delta_{0} /(1-2 \beta)}\right)\left[\|f\|_{\infty} C_{2} \varepsilon_{n}\left(a_{n}\right)+\Delta_{n} C_{2} \varepsilon_{n}\left(a_{n}\right)+\Delta_{n}\right] \\
\leqslant C C_{\omega}\left(k a_{n}^{2 \delta_{0} /(1-2 \beta)}\right)\left[\varepsilon_{n}\left(a_{n}\right)+\Delta_{n}\right]
\end{aligned}
$$


с некоторой константой $C>0$. Тогда в силу выбора $\Delta_{n}$ и $a_{n}$ мы имеем $P\left(E_{n, a_{n}}^{(2)}(\varepsilon)\right) \rightarrow 1$ при $n \rightarrow \infty$.

Заметим, что на событии $E_{n}^{(1)} \cap E_{n, a_{n}}^{(2)}(\varepsilon)$ выполнено неравенство

$$
\begin{aligned}
\sup _{x \notin \mathscr{M}_{\delta(\varepsilon)}} \omega\left(\widehat{f}_{n}(x), x\right) & \leqslant \sup _{x \notin \mathscr{M}_{\delta(\varepsilon)}} \omega(f(x), x)+\frac{\varepsilon}{3} \leqslant \sup _{x \in W_{a_{n}}} \omega(f(x), x)-\frac{2 \varepsilon}{3} \\
& \leqslant \sup _{x \in W_{a_{n}}} \omega\left(\widehat{f}_{n}(x), x\right)-\frac{\varepsilon}{3}
\end{aligned}
$$

из которого следует, что

$$
\sup _{x \in W_{a_{n}}} \omega\left(\widehat{f}_{n}(x), x\right)=\sup _{x \in \mathscr{M}_{\delta(\varepsilon)}} \omega\left(\widehat{f}_{n}(x), x\right) .
$$

Для всех $\tau \in \mathscr{M}$ и всех $x \in \mathscr{M}_{\delta(\varepsilon)}$ таких, что $|x-\tau|<\delta(\varepsilon)$, мы имеем

$$
\begin{aligned}
& \omega\left(\widehat{f}_{n}(x), x\right)-\omega(f(\tau), \tau) \\
& =\omega\left(\widehat{f}_{n}(\tau), \tau\right)-\omega(f(\tau), \tau)+\omega(f(x), x)-\omega(f(\tau), \tau) \\
& \quad+\left[\omega\left(\widehat{f}_{n}(x), x\right)-\omega\left(\widehat{f}_{n}(\tau), \tau\right)\right]-[\omega(f(x), x)-\omega(f(\tau), \tau)] .
\end{aligned}
$$

Следовательно, на событии $E_{n}^{(1)} \cap E_{n, a_{n}}^{(2)}(\varepsilon)$ выполнены равенства

$$
\begin{gathered}
\sup _{x \in W_{a_{n}}} \omega\left(\widehat{f}_{n}(x), x\right)-\sup _{x \in W_{a_{n}}} \omega(f(x), x)=\sup _{x \in \mathscr{M}_{\delta(\varepsilon)}} \omega\left(\widehat{f}_{n}(x), x\right)-\sup _{\tau \in \mathscr{M}} \omega(f(\tau), \tau) \\
=\sup _{\tau \in \mathscr{M}}\left[\omega\left(\widehat{f}_{n}(\tau), \tau\right)-\omega(f(\tau), \tau)+\sup _{|x-\tau| \leqslant \delta(\varepsilon)} \omega(f(x), x)-\omega(f(\tau), \tau)\right. \\
\quad+\sup _{|x-\tau| \leqslant \delta(\varepsilon)}\left(\left[\omega\left(\widehat{f}_{n}(x), x\right)-\omega\left(\widehat{f}_{n}(\tau), \tau\right)\right]\right. \\
\quad-[\omega(f(x), x)-\omega(f(\tau), \tau)])] \\
=\sup _{\tau \in \mathscr{M}}\left[\omega\left(\widehat{f}_{n}(\tau), \tau\right)-\omega(f(\tau), \tau)+r_{n}(\tau, \delta(\varepsilon))\right],
\end{gathered}
$$

где

$r_{n}(\tau, \delta(\varepsilon)):=\sup _{|x-\tau| \leqslant \delta(\varepsilon)}\left(\left[\omega\left(\widehat{f}_{n}(x), x\right)-\omega\left(\widehat{f}_{n}(\tau), \tau\right)\right]-[\omega(f(x), x)-\omega(f(\tau), \tau)]\right)$

(последнее равенство в (20) верно, поскольку $\sup _{|x-\tau| \leqslant \delta(\varepsilon)} \omega(f(x), x)-$ $\omega(f(\tau), \tau)=0)$. Используя условие $(\mathrm{A} 1)$, мы можем оценить $\left|r_{n}\right|$ следующим образом:

$$
\begin{aligned}
& \sup _{\tau \in \mathscr{M}}\left|r_{n}(\tau, \delta(\varepsilon))\right|=\sup _{\tau \in \mathscr{M}} \sup _{x \in \mathscr{M}_{\delta(\varepsilon)},|x-\tau| \leqslant \delta(\varepsilon)} \mid \int_{0}^{1} \frac{\partial}{\partial u} \omega\left(\lambda \widehat{f}_{n}(s)\right. \\
& \quad+(1-\lambda) f(s), s)\left.d \lambda\left(\widehat{f}_{n}(s)-f(s)\right)\right|_{s=\tau} ^{s=x} \mid \\
& =\sup _{\tau \in \mathscr{M}} \sup _{x \in \mathscr{M}_{\delta(\varepsilon)},|x-\tau| \leqslant \delta(\varepsilon)}\left|\int_{0}^{1} \nabla g_{n}(\mu x+(1-\mu) \tau) d \mu(x-\tau)\right| \\
& \leqslant \sup _{\tau \in \mathscr{M}} \sup _{x \in \mathscr{M}_{\delta(\varepsilon)}}\left|\nabla g_{n}(x)\right| \delta(\varepsilon),
\end{aligned}
$$


где $\nabla g$ обозначает градиент функции $g$ и для $s \in \mathscr{M}_{\delta(\varepsilon)}$

$$
g_{n}(s):=\int_{0}^{1} \frac{\partial}{\partial u} \omega\left(\lambda \widehat{f}_{n}(s)+(1-\lambda) f(s), s\right) d \lambda\left(\widehat{f}_{n}(s)-f(s)\right) .
$$

Рассмотрим $\nabla g_{n}(s)$ для любого $s \in \mathscr{M}_{\delta(\varepsilon)}$ :

$$
\begin{aligned}
\nabla g_{n}(s)= & \int_{0}^{1} \frac{\partial^{2}}{\partial u^{2}} \omega\left(\lambda \widehat{f}_{n}(s)+(1-\lambda) f(s), s\right) d \lambda\left(\widehat{f}_{n}(s)-f(s)\right)^{2} \\
& +\int_{0}^{1} \frac{\partial}{\partial u} \omega\left(\lambda \widehat{f}_{n}(s)+(1-\lambda) f(s), s\right) d \lambda\left(\nabla \widehat{f}_{n}(s)-\nabla f(s)\right) \\
& +\int_{0}^{1} \frac{\partial^{2}}{\partial u \partial t} \omega\left(\lambda \widehat{f}_{n}(s)+(1-\lambda) f(s), s\right) d \lambda\left(\widehat{f}_{n}(s)-f(s)\right) .
\end{aligned}
$$

Тогда $\delta(\varepsilon) \rightarrow 0$ для $s \in \overline{\operatorname{conv}\left(\mathscr{M}_{\delta(\varepsilon)}\right)} \subset \mathscr{K} \subset W_{a_{n}}$. Таким образом, для всех $\lambda \in[0,1]$ и всех $s \in \overline{\operatorname{conv}\left(\mathscr{M}_{\delta(\varepsilon)}\right)}$

$$
\begin{aligned}
& \lambda\left(1-C_{2} \varepsilon_{n}\left(a_{n}\right)\right) \tilde{f}_{n}(s)+(1-\lambda) f(x) \\
& \quad \leqslant \lambda \widehat{f}_{n}(s)+(1-\lambda) f(s) \leqslant \lambda\left(1+C_{2} \varepsilon_{n}\left(a_{n}\right)\right) \tilde{f}_{n}(s)+(1-\lambda) f(x), \\
& \lambda\left(1-C_{2} \varepsilon_{n}\left(a_{n}\right)\right)\left(f(s)-\Delta_{n}\right)+(1-\lambda) f(x) \leqslant \lambda \widehat{f}_{n}(s)+(1-\lambda) f(s) \\
& \quad \leqslant \lambda\left(1+C_{2} \varepsilon_{n}\left(a_{n}\right)\right)\left(f(s)+\Delta_{n}\right)+(1-\lambda) f(x), \\
& f(s)-\varepsilon_{n}^{*} \leqslant f(s)-\lambda\left(\|f\|_{\infty} C_{2} \varepsilon_{n}\left(a_{n}\right)+\Delta_{n}-C_{2} \varepsilon_{n}\left(a_{n}\right) \Delta_{n}\right) \\
& \quad \leqslant \lambda \widehat{f}_{n}(s)+(1-\lambda) f(s) \\
& \quad \leqslant f(s)+\lambda\left(\|f\|_{\infty} C_{2} \varepsilon_{n}\left(a_{n}\right)+\Delta_{n}+C_{2} \varepsilon_{n}\left(a_{n}\right) \Delta_{n}\right) \leqslant f(s)+\varepsilon_{n}^{*}
\end{aligned}
$$

при $n \rightarrow \infty$, где $\varepsilon_{n}^{*}=O_{P}\left(\varepsilon_{n}\left(a_{n}\right)+\Delta_{n}\right)$. Тогда в силу (A1) мы получаем

$$
\begin{aligned}
\sup _{x \in \mathscr{K}}\left|\nabla g_{n}(x)\right| \leqslant C_{3} & \left(\sup _{x \in \mathscr{K}}\left|\widehat{f}_{n}(s)-f(s)\right|^{2}+\sup _{x \in \mathscr{K}}\left|\widehat{f}_{n}(s)-f(s)\right|\right. \\
& \left.+\sup _{x \in \mathscr{K}}\left|\nabla \widehat{f}_{n}(s)-\nabla f(s)\right|\right)
\end{aligned}
$$

с некоторой конечной константой $C_{3}>0$. На событии $E_{n}^{(1)} \cap E_{n, a_{n}}^{(2)}(\varepsilon)$ выполнены неравенства $\sup _{x \in \mathscr{K}}\left|\widetilde{f}_{n}(x)-f(x)\right| \leqslant \Delta_{n}, \sup _{x \in \mathscr{K}} \mid \nabla \widetilde{f}_{n}(x)-$ $\nabla f(x) \mid \leqslant \Delta_{n}$, откуда, используя предложение 1.1 из [5] с функцией $\Psi=$ $I_{D_{a_{0}}}$ и $\mathscr{K} \subset D_{a_{0}}$ для некоторого $a_{0}>0$, получаем

$$
\sup _{x \in \mathscr{K}}\left|\widehat{f}_{n}(x)-\tilde{f}_{n}(x)\right| \leqslant C_{4} \sqrt{\frac{\left|\ln h_{n}^{d}\right|}{n h_{n}^{d}}}
$$

при $n \rightarrow \infty$. Еще нам нужна следующая стандартная лемма.

Лемма 3. Предположим, что ядро $K$ - дважды непрерьвно дифферениируемая на ограниченном носителе функция, и пусть $f$ дифференцируема. Пусть $n h_{n}^{d+4} \rightarrow \infty$ nри $n \rightarrow \infty$. Тогда с некоторой ограниченной константой $C_{5}$ выполняется следующее неравенство:

$$
\mathbf{E} \sup _{x \in \mathscr{K}}\left|\nabla \widehat{f}_{n}(x)-\nabla \tilde{f}_{n}(x)\right|^{2} \leqslant \frac{C_{5}^{2}}{n h_{n}^{d+4}} \quad \text { npu } n \rightarrow \infty .
$$


Отсюда вытекает, что на некотором событии $E_{n}^{(3)}$ таком, что $P\left(E_{n}^{(3)}\right) \rightarrow 1$ при $n \rightarrow \infty$, мы имеем

$$
\sup _{x \in \mathscr{K}}\left|\nabla \widehat{f}_{n}(x)-\nabla \widetilde{f}_{n}(x)\right| \leqslant \frac{C_{5}}{\sqrt{n h_{n}^{d+4}}} .
$$

Тогда на событии $E_{n}^{(1)} \cap E_{n, a_{n}}^{(2)}(\varepsilon) \cap E_{n}^{(3)}$ верна оценка

$$
\sup _{x \in \mathscr{K}}\left|\nabla g_{n}(x)\right| \leqslant C_{6}\left(\Delta_{n}+\frac{1}{\sqrt{n h_{n}^{d+4}}}\right),
$$

из которой на событии $E_{n}^{(1)} \cap E_{n, a_{n}}^{(2)}(\varepsilon) \cap E_{n}^{(3)}$ вытекает

$$
\sup _{\tau \in \mathscr{K}}\left|r_{n}(\delta(\varepsilon), \tau)\right| \leqslant C_{6} \delta(\varepsilon)\left(\Delta_{n}+\frac{1}{\sqrt{n h_{n}^{d+4}}}\right)
$$

и, как следствие,

$$
\begin{aligned}
& \left|\ln h_{n}\right|\left|\sup _{x \in W_{a_{n}}} \omega\left(\widehat{f}_{n}(x), x\right)-\sup _{x \in W_{a_{n}}} \omega(f(x), x)\right| \\
& \quad \leqslant\left|\ln h_{n}\right|\left[\Delta_{n}+C_{5} \sqrt{\frac{\left|\ln h_{n}^{d}\right|}{n h_{n}^{d}}}+C_{6} \delta(\varepsilon)\left(\Delta_{n}+\frac{1}{\sqrt{n h_{n}^{d+4}}}\right)\right] .
\end{aligned}
$$

Пусть $\varepsilon=\varepsilon_{n} \rightarrow 0$ таково, что $\delta\left(\varepsilon_{n}\right) \leqslant O\left(h_{n}^{2}\right)$ при $n \rightarrow \infty$. Поскольку $\left|\ln h_{n}\right| \Delta_{n} \stackrel{P}{\longrightarrow} 0$ и $\left|\ln h_{n}\right|^{3 / 2} / \sqrt{n h_{n}^{d}} \rightarrow 0$, то при $n \rightarrow \infty$

$$
\left|\ln h_{n}\right|\left|\sup _{x \in W_{a_{n}}} \omega\left(\widehat{f}_{n}(x), x\right)-\sup _{x \in W_{a_{n}}} \omega(f(x), x)\right| \stackrel{P}{\longrightarrow} 0,
$$

и, как следствие, мы можем заменить $A_{1 / h_{n}}$ на $\widehat{B}_{n}$ в $(19)$.

3. Чтобы заменить $\omega(f(x), x) f^{-1 / 2}(x)$ на $\omega\left(\widehat{f}_{n}(x), x\right) \hat{f}_{n}^{-1 / 2}(x)$ в (19), нам надо показать, что

$$
\begin{aligned}
\sqrt{n h_{n}^{d}\left|\ln h_{n}^{d}\right|} \sup _{x \in D_{a_{n}}} \mid\left[\omega\left(\widehat{f}_{n}(x), x\right) \widehat{f}_{n}^{-1 / 2}(x)-\omega(f(x), x) f^{-1 / 2}(x)\right] \\
\quad \times\left[\widehat{f}_{n}(x)-\widetilde{f}_{n}(x)\right] \mid \\
\leqslant\left|\ln h_{n}\right| O_{P}(1) \sup _{x \in D_{a_{n}}}\left|\frac{\omega\left(\widehat{f}_{n}(x), x\right) \widehat{f}_{n}^{-1 / 2}(x)-\omega(f(x), x) f^{-1 / 2}(x)}{\omega(f(x), x) f^{-1 / 2}(x)}\right| \stackrel{P}{\longrightarrow} 0
\end{aligned}
$$

при $n \rightarrow \infty$. С этой целью рассмотрим

$$
\begin{aligned}
& \omega\left(\widehat{f}_{n}(x), x\right) \widehat{f}_{n}^{-1 / 2}(x)-\omega(f(x), x) f^{-1 / 2}(x) \\
& =\int_{0}^{1}\left[\frac{\partial}{\partial u} \omega(u, x) u^{-1 / 2}\right. \\
& \left.\quad-0.5 \omega(u, x) u^{-3 / 2}\right]\left.\right|_{u=\lambda \widehat{f}_{n}(x)+(1-\lambda) f(x)} d \lambda\left(\widehat{f}_{n}(x)-f(x)\right) .
\end{aligned}
$$


Тогда на событии $E_{n}^{(1)}$

$$
\begin{aligned}
& \sup _{x \in D_{a_{n}}}\left|\frac{\omega\left(\widehat{f}_{n}(x), x\right) \widehat{f}_{n}^{-1 / 2}(x)-\omega(f(x), x) f^{-1 / 2}(x)}{\omega(f(x), x) f^{-1 / 2}(x)}\right| \\
& \leqslant C_{7} \sup _{x \in D_{a_{n}}} \frac{\left|\widehat{f}_{n}(x)-f(x)\right|}{\omega(f(x), x) f(x)} \\
& \leqslant C_{7} a_{n} \frac{\|f\|_{\infty} C_{2} \varepsilon_{n}\left(a_{n}\right)+\Delta_{n}+C_{2} \Delta_{n} \varepsilon_{n}\left(a_{n}\right)}{\inf _{s \in D_{a_{n}}} \omega(f(s), s)} \\
& \leqslant C_{8} a_{n}^{1+\delta_{0}}\left[\varepsilon_{n}\left(a_{n}\right)+\Delta_{n}\right],
\end{aligned}
$$

где $C_{7}, C_{8}$ - положительные константы. Выбор $\Delta_{n}$ и $a_{n}$ гарантирует нужный предельный результат.

4. Наконец, смещением можно пренебречь, если при $n \rightarrow \infty$

$$
B_{n} \sqrt{n h_{n}^{d}} \sup _{x \in D_{a_{n}}} \omega(f(x), x) f^{-1 / 2}(x) \Delta_{n} \leqslant C_{9} \sqrt{n h_{n}^{d}\left|\ln h_{n}\right|} c a_{n}^{\beta} \Delta_{n} \stackrel{P}{\longrightarrow} 0,
$$

где $C_{9}>0$ - константа, что завершает доказательство теоремы 5 .

3 а м е ч а н и е. Из условия на смещение в теореме 5 и условий $(\mathrm{H} 1),(\mathrm{H} 2)$ следует, что если $\Delta_{n}=O_{P}\left(h_{n}^{r}\right)$ с $r>0$, то $r$ должно быть больше 2 , и тогда остальные условия на $a_{n}$ и $\Delta_{n}$ должны выполняться.

6.2. Примеры. 1. Пусть $\omega(u, x)=\Psi(x) \sqrt{u}$, где $\Psi$ удовлетворяет условиям (W.a)-(W.c) и (WD.a $)_{\beta}$. Поскольку

$$
\begin{gathered}
\frac{\partial \omega(u, x)}{\partial u}=0.5 \Psi(x) u^{-1 / 2}, \quad \frac{\partial^{2} \omega(u, x)}{\partial u^{2}}=-0.25 \Psi(x) u^{-3 / 2}, \\
\frac{\partial^{2} \omega(u, x)}{\partial u \partial x}=0.5 \nabla \Psi(x) u^{-1 / 2}
\end{gathered}
$$

для $u \in \mathbf{R}_{+}, x \in \mathbf{R}^{d}$, мы можем записать условие (A1) в виде

$$
\sup _{x \in \mathscr{K}}\left[\Psi(x) f(x)^{-3 / 2}+\Psi(x) f(x)^{-1 / 2}+|\nabla \Psi(x)| f(x)^{-1 / 2}\right]<\infty,
$$

условие $(\mathrm{A} 2)$ работает с $C_{\omega}(a)=C a$ для некоторой константы $C>0$ и всех достаточно больших $a>0$, а условие (А3) выполнено для $\Psi$ такого, что $\inf _{x \in D_{a}} \Psi(x) \geqslant C_{0} a^{0.5-\delta_{0}}$.

Такой выбор весовой функции был рассмотрен в [4] для одномерного случая. Были предложены похожие условия на $\Delta_{n}$, супремум брался по $\mathbf{R}$ вместо $\widehat{D}_{a_{n}}$. Доказательство в [4] было более специализированным.

2. Пусть $\omega(u, x)=u^{0.5-\beta}$ с некоторым $\beta \in(0,1 / 2)$. Поскольку

$$
\begin{gathered}
\frac{\partial \omega(u, x)}{\partial u}=(0.5-\beta) u^{-0.5-\beta}, \quad \frac{\partial^{2} \omega(u, x)}{\partial u^{2}}=-(0.5-\beta)(0.5+\beta) u^{-3 / 2-\beta}, \\
\frac{\partial^{2} \omega(u, x)}{\partial u \partial x}=0
\end{gathered}
$$


для $u \in \mathbf{R}_{+}, x \in \mathbf{R}^{d}$, условие (А1) выполнено автоматически в силу $\mathscr{K} \subset D_{a_{0}}$ для некоторого конечного $a_{0}>0$, условие (А2) работает с $C_{\omega}(a)=C a^{-(0.5+\beta)}$ для некоторой константы $C>0$ и всех достаточно больших $a>0$, а условие (А3) выполнено с $C_{0}=1$ и $\delta_{0}=0.5-\beta$.

Такой выбор весовой функции был рассмотрен в [3] для одномерного случая. Условия на $a_{n}$ и $\Delta_{n}$ в [3] являются особым случаем наших условий. Доказательство в [3] было более сложным и специализированным.

Автор благодарит профессоров Владимира Колчинского и Ильдара Ибрагимова за поощрение и полезные разговоры по теме данной работы. Автор также признательна рецензентам, чьи замечания привели к улучшению настоящей статьи.

\section{СПИСОК ЛИТЕРАТУРЫ}

1. Bickel P. J., Rosenblatt M. On some global measures of the deviations of density function estimates. - Ann. Statist., 1973, v. 1, p. 1071-1095.

2. Гихман И. И., Скороход А.В. Введение в теорию случайных процессов. М.: Наука, 1965, 655 с.

3. Giné E., Koltchinskii V., Sakhanenko L. Convergence in distribution of selfnormalized sup-norms of kernel density estimators. High Dimensional Probability III. Ed. by J. Hoffmann-Jörgensen et al. Basel: Birkhäuser, 2003, p. 241-253.

4. Giné E., Koltchinskii V., Sakhanenko L. Kernel density estimators: convergence in distribution for weighted sup-norms. - Probab. Theory Related Fields, 2004, v. 130, № 2, p. 167-198.

5. Giné E., Koltchinskii V., Zinn J. Weighted uniform consistency of kernel density estimators. - Ann. Probab., 2004, v. 32, № 3B, p. 2570-2605.

6. Komlós J., Major P., Tusnády G. An approximation of partial sums of independent RV's and the sample DF. I. - Z. Wahrscheinlichtkeitstheor. verw. Geb., 1975, v. 32, p. $111-131$.

7. Konakov V.D., Piterbarg V.I. On the convergence rate of maximal deviation distribution for kernel regression estimates. - J. Multivariate Anal., 1984, v. 15, p. $279-294$

8. Ledoux M., Talagrand M. Probability on Banach Spaces. New York: Springer-Verlag, 1991, $480 \mathrm{p}$.

9. Лифиии М. А. Гауссовские случайные функции. Киев: ТВиМС, 1995, 246 с.

10. Pickands J. III. Upcrossing probabilities for stationary Gaussian processes. - Trans. Amer. Math. Soc., 1969, v. 145, p. 51-73.

11. Piterbarg V. Asymptotic Methods in the Theory of Gaussian Processes and Fields. Providence, RI: Amer. Math. Soc., 1996, 206 p. (Transl. Math. Monogr., 1995, v. 148.)

12. Rio E. Local invariance principles and their application to density estimation. Probab. Theory Related Fields, 1994, v. 98, № 1, p. 21-45.

13. van der Vaart A., Wellner J. A. Weak Convergence and Empirical Processes with Applications to Statistics. New York: Springer-Verlag, 1996, 508 p.

Поступила в редакцию 04.I.2012

Исправленный вариант 11.V.2014 